\title{
Multiscale simulations in simple metals: A density-functional-based methodology
}

\author{
Nicholas Choly, ${ }^{1, *}$ Gang Lu, ${ }^{1, \dagger}$ Weinan E, ${ }^{2}$ and Efthimios Kaxiras ${ }^{1}$ \\ ${ }^{1}$ Division of Engineering and Applied Sciences, Harvard University, Cambridge, Massachusetts 02138, USA \\ ${ }^{2}$ Department of Mathematics and Program in Applied and Computational Mathematics, Princeton University, \\ Princeton, New Jersey 08544, USA
}

(Received 17 April 2004; revised manuscript received 25 October 2004; published 3 March 2005)

\begin{abstract}
We present a formalism for coupling a density-functional-theory-based quantum simulation to a classical simulation for the treatment of simple metallic systems. The formalism is applicable to multiscale simulations in which the part of the system requiring quantum-mechanical treatment is spatially confined to a small region. Such situations often arise in physical systems where chemical interactions in a small region can affect the macroscopic mechanical properties of a metal. We describe how this coupled treatment can be accomplished efficiently, and we present a coupled simulation for a bulk aluminum system.
\end{abstract}

DOI: $10.1103 /$ PhysRevB.71.094101

PACS number(s): 71.15.Mb, 71.15.Dx, 62.20.Mk

\section{INTRODUCTION}

In performing computer simulations of complex physical systems, a premium is placed on accuracy and efficiency. Typically, one of these qualities can be improved at the expense of the other. In recent years, a new approach has emerged that addresses a class of problems in which important small-length-scale phenomena are confined to a small region of the system but can have an impact on the behavior over a much larger scale. A typical case is the tip of a crack, where localized chemical reactions may affect the strength of interatomic bonding, which in turn can influence in a dramatic way the macroscopic mechanical properties of the solid. Such problems fall under the rubric of "multiscale" phenomena, requiring a treatment that addresses important aspects at each scale. ${ }^{1}$ The novel feature of this type of simulation is to use an accurate but computationally demanding method to treat the region of the system in which small-length-scale degrees of freedom are important, and a faster but less accurate method with the smalllength-scale physics "coarse-grained" to treat the rest of the system.

Multiscale approaches rely on successfully coupling the two (or more) regions involved, which is referred to as seamless coupling. There is no single notion as to what constitutes a seamless coupling, but generally the coupling should be accomplished in such a way that the fictitious boundary between the two regions, which only exists in the coupled simulation and not in the real system, does not introduce any physical consequences. For instance, recently several papers have dealt with the issue of ensuring that phonons are not reflected by the boundary between the two coupling methods. ${ }^{2,3}$ In the consummate multiscale method, the resulting energetics or dynamics is indistinguishable from what would result from a calculation with the accurate method applied to the entire system. This ideal would be achieved only if the two simulation methods involved were seamlessly matched at the boundary, and further, only if the part of the system treated by the faster, less accurate method was indeed free of important small-scale physics. Another important but obvious characteristic of a good multiscale method is that the computational overhead of performing a coupled simulation is not significant. More specifically, the computation time for the coupled simulation should be on the order of computation time required for the accurate method to treat the small detailed region, since the time required for the less accurate method to treat the rest of the system is typically several orders smaller; nothing is gained if the coupling is so costly that the coupled method takes as long as using the accurate method to treat the whole system. When the approach requires coupling a quantum-mechanical method to a classical method, additional complications arise because of the presence of electronic degrees of freedom in the quantummechanical region; thus boundary conditions on the electron wave functions must be imposed at the interface between the regions. Density-functional theory (DFT) provides a significant simplification over more direct quantum-mechanical methods in that the calculation of ground state energies and forces requires the minimization of a functional of the electron density $\rho(\mathbf{r})$ only. ${ }^{4}$ Thus, in principle, boundary conditions need only be imposed on $\rho(\mathbf{r})$. This statement only applies to the formulation of the problem that does not invoke the explicit calculation of electronic wave functions (the most common way of implementing DFT actually does involve individual electronic wave functions, the so-called Kohn-Sham orbitals ${ }^{5}$ ). Coupling an approximate DFT calculation that is based on the electronic density alone to a classical interatomic potential should be more straightforward than coupling an orbital-based quantum-mechanical method to a classical method.

The present article describes a formalism for concurrently coupling a system consisting of two regions, one treated with density-functional theory (without invoking electronic wave functions) and the other with a classical interatomic potential. Due to the type of approximations involved, the present approach is particularly well suited for simple metallic systems; we emphasize, however, that this is not an inherent limitation of our approach, but rather a limitation imposed by the shortcomings of the methodologies employed for the treatment of the various parts of the system, and if these are eliminated the approach could be generally applicable. In Sec. II, other methodologies for coupling multiple simulation approaches and their relevance to the present methods are 
discussed. In Sec. III, the formalism of the present class of coupling methods is established. In Sec. IV, details of implementing the methods and achieving efficiency are presented and some tests of the method are reported in Sec. V. Finally we conclude in Sec. VI with a discussion of the results of the tests.

\section{BACKGROUND}

A large number of concurrent multiscale methods ${ }^{6-8}$ approach the problem of coupling two different simulation methods by writing the energy of the whole system as

$$
E[\mathrm{I}+\mathrm{II}]=E_{1}[\mathrm{I}]+E_{2}[\mathrm{II}]+E^{\mathrm{int}}[\mathrm{I}, \mathrm{II}],
$$

where here and throughout the article, I refers to the small region where detailed physics are relevant, and II refers to the rest of the system. $E_{1}[\mathrm{I}]$ represents the energy of region I with region II providing appropriate boundary conditions, $E_{2}[\mathrm{II}]$ represents the energy of region II in the same sense, and $E[\mathrm{I}+\mathrm{II}]$ represents the total energy of the combined system. Equation (1) expresses the total energy of the system as the energy of region I evaluated with the accurate simulation method 1, plus the energy of region II evaluated with the faster simulation method 2, plus an energy of interaction between the two subsystems. Typically, the crux of a multiscale method lies in its handling of $E^{\text {int }}$. Although tautological, Eq. (1) can be rearranged to yield an expression for the interaction energy:

$$
E^{\mathrm{int}}[\mathrm{I}, \mathrm{II}]=E[\mathrm{I}+\mathrm{II}]-E_{1}[\mathrm{I}]-E_{2}[\mathrm{II}] .
$$

This expression contains no new content, and merely serves to define $E^{\text {int }}[\mathrm{I}, \mathrm{II}]$, but nevertheless provides direction towards its calculation.

The quantum-mechanical/molecular-mechanical (QM/ MM) methods are designed to achieve a goal similar to that of the present method, namely, the coupling of a quantummechanical simulation with classical potentials, but in the context of covalently bonded organic molecules. In such systems, bonds are localized and typically can be associated with two atoms at either end. The strategy often employed in QM and MM methods to couple quantum mechanics to molecular mechanics is as follows: ${ }^{9}$ the system is divided into QM and MM regions with a boundary that cuts across covalent bonds; $E_{Q M}$ is evaluated for the QM subsystem, plus additional "link atoms" placed on the MM side of the severed covalent bonds to mimic the system removed from the QM region; $E_{M M}$ is evaluated for the MM subsystem without the link atoms; and $E_{Q M / M M}$ consists of energy terms such as bond-bending terms that are left out of $E_{Q M}+E_{M M}$. A similar methodology was developed by Broughton et $a l^{8}$ for quantum-classical coupling in silicon, the prototypical covalently bonded bulk material. Such approaches rely on a somewhat artificial partitioning of the total energy (e.g., into bond-bending and bondstretching terms), and hence lack a definition that could be readily generalized. But due to the locality of physics in covalently bonded systems for which QM and $\mathrm{MM}$ methods are appropriate, errors introduced at the QM/MM boundary typically do not manifest themselves throughout the system.

In metallic systems, however, the situation is quite different. Bonds are not localized or associated with a distinct pair of atoms. The embedded-atom picture ${ }^{10,11}$ provides a more apt description of the situation. In the embedded-atom picture of a simple metallic system, the density of the system is approximately the sum of charge densities of isolated atoms, and the energetic contribution of an individual atom to the system energy is approximately the embedding energy of the atom in a homogeneous electron gas. This picture, in various forms, ${ }^{11-13}$ has been used to great effect to create classical pair functionals for metals. The success of these potentials in capturing the energetics of simple metallic systems, combined with their foundation on density-functional-theory arguments, makes them ideal candidates for evaluating $E_{2}[\mathrm{II}]$ in the present formalism.

The notions of the embedded-atom method can be extended to describe the energetics of a metallic region (region I) within another metallic region (region II); region I is embedded within region II. The exact nature of the embedding can be formally written in the manner of Eq. (1) with density-functional-theory arguments. We first decide which ions will be associated with region I and which will be in region II, and we will denote those sets of nuclear coordinates by $\mathbf{R}^{\mathrm{I}}$ and $\mathbf{R}^{\mathrm{II}}$. We denote the set of all nuclei with $\mathbf{R}^{\text {tot }} \equiv \mathbf{R}^{\mathrm{I}} \cup \mathbf{R}^{\mathrm{II}}$. According to the Hohenberg-Kohn theorem, the total system energy, within the Born-Oppenheimer approximation, is given by minimizing a functional of the total charge density:

$$
E\left[\mathbf{R}^{\mathrm{tot}}\right]=\min _{\rho} E_{\mathrm{DFT}}\left[\rho^{\mathrm{tot}} ; \mathbf{R}^{\mathrm{tot}}\right] .
$$

In order to be explicit, by $E_{\mathrm{DFT}}[\rho ;\{\mathbf{R}\}]$ we mean

$$
\begin{aligned}
E_{\mathrm{DFT}}[\rho ; \mathbf{R}] \equiv & T_{\mathrm{s}}[\rho]+E_{\mathrm{H}}[\rho]+E_{\mathrm{xc}}[\rho] \\
& +\sum_{i} \int \rho(\mathbf{r}) V_{\mathrm{psp}}\left(\mathbf{r}-\mathbf{R}_{i}\right) \mathrm{dr}+\sum_{i<j} \frac{Z_{i} Z_{j}}{\left|\mathbf{R}_{i}-\mathbf{R}_{j}\right|},
\end{aligned}
$$

where $T_{\mathrm{s}}$ is the noninteracting kinetic energy functional, $E_{\mathrm{H}}$ is the Hartree energy, $E_{\mathrm{xc}}$ is the exchange-correlation energy, and $V_{\mathrm{psp}}$ is the ionic pseudopotential. Thus $E_{\mathrm{DFT}}$ represents the combined electronic and ion-ion (Madelung) energy.

If $\rho^{\text {tot }}$ is partitioned into two subdensities, $\rho^{\mathrm{I}}$ and $\rho^{\mathrm{II}}$, such that $\rho^{\text {tot }}=\rho^{\mathrm{I}}+\rho^{\mathrm{II}}$, then the $E_{\mathrm{DFT}}$ can be partitioned:

$$
\begin{aligned}
E_{\mathrm{DFT}}\left[\rho^{\mathrm{tot}} ; \mathbf{R}^{\mathrm{tot}}\right]= & E_{\mathrm{DFT}}\left[\rho^{\mathrm{I}} ; \mathbf{R}^{\mathrm{I}}\right]+E_{\mathrm{DFT}}\left[\rho^{\mathrm{II}} ; \mathbf{R}^{\mathrm{II}}\right] \\
& +E^{\mathrm{int}}\left[\rho^{\mathrm{I}}, \rho^{\mathrm{II}} ; \mathbf{R}^{\mathrm{I}}, \mathbf{R}^{\mathrm{II}}\right],
\end{aligned}
$$

where $E^{\text {int }}$ is defined as in Eq. (2). By varying the total energy with respect to $\rho^{\mathrm{I}}$, we find that the potential acting upon $\rho^{\mathrm{I}}$ is equal to the sum of the potential from region I alone, plus an embedding potential $V_{\text {emb }}(\mathbf{r})$ that completely represents the effect of region II upon region I: 


$$
\begin{gathered}
\frac{\delta E_{\mathrm{DFT}}\left[\rho^{\mathrm{tot}} ; \mathbf{R}^{\mathrm{tot}}\right]}{\delta \rho^{I}}=\frac{\delta E_{\mathrm{DFT}}\left[\rho^{\mathrm{I}} ; \mathbf{R}^{\mathrm{I}}\right]}{\delta \rho^{\mathrm{I}}}+V_{\mathrm{emb}}(\mathbf{r}), \\
V_{\mathrm{emb}}(\mathbf{r}) \equiv \frac{\delta E^{\mathrm{int}}\left[\rho^{\mathrm{I}}, \rho^{\mathrm{II}} ; \mathbf{R}^{\mathrm{I}}, \mathbf{R}^{\mathrm{II}}\right]}{\delta \rho^{\mathrm{I}}} .
\end{gathered}
$$

By using different approximations for the terms in Eq. (6), different coupled methods are obtained. Wesolowski and Warshel, ${ }^{14}$ building on the formalism of Cortona, ${ }^{15}$ used this partitioning to describe an efficient DFT method. In their scheme $E[\mathrm{I}]$ and $E[\mathrm{II}]$ are treated with Kohn-Sham DFT, but $E^{\text {int }}$ is evaluated with "orbital-free" density functional theory (OF-DFT), i.e., pure density-functional theory in which the Kohn-Sham orbitals are not used and the noninteracting kinetic energy is approximated with an explicit functional of the density. ${ }^{16-20}$ This allows $E[\mathrm{I}]$ and $E[\mathrm{II}]$ to be alternately minimized in the embedding potential of the other. Govind et al. ${ }^{7}$ utilized the partitioning of Eq. (5) to obtain a quantum chemistry (QC)-DFT coupled method. There $E_{1}[\mathrm{I}]$ was calculated with QC, $E_{2}[\mathrm{II}]$ with DFT, and again $E^{\text {int }}$ was based on OF-DFT. They used this method to explore the electronic structure of molecules adsorbed on metal surfaces. Recently Klüner et $a .^{21}$ have extended this formalism to treat adsorbed molecules in their excited state.

\section{FORMALISM}

The present method follows in the same vein as the last few examples, to achieve a DFT-classical potential coupling. The general idea of the present methods is as follows. $E_{1}[\mathrm{I}]$ is to be calculated with DFT. $E_{2}[\mathrm{II}]$ is calculated via a classical potential. A choice can be made for the calculation of $E^{\text {int }}$, which results in distinct coupling methods, which we examine in detail below.

\section{A. Classical interaction energy}

$E^{\text {int }}$ can be calculated using the classical potential:

$$
E^{\mathrm{int}}[\mathrm{I}, \mathrm{II}]=E_{\mathrm{cl}}[\mathrm{I}+\mathrm{II}]-E_{\mathrm{cl}}[\mathrm{I}]-E_{\mathrm{cl}}[\mathrm{II}] .
$$

Although this interaction energy is intended to represent the same DFT interaction energy that appears in Eq. (5), it is not contradictory to use the classical potential to evaluate it, since the classical potential energy, evaluated for a given ionic configuration $\mathbf{R}$, can be viewed as an approximation to the DFT functional that has been minimized with respect to the density; that is,

$$
E_{\mathrm{cl}}[\mathbf{R}] \simeq \min _{\rho} E_{\mathrm{DFT}}[\rho, \mathbf{R}] .
$$

This choice of interaction energy results in a total energy of

$$
E\left[\mathbf{R}^{\mathrm{tot}}\right]=E_{\mathrm{cl}}\left[\mathbf{R}^{\mathrm{tot}}\right]-E_{\mathrm{cl}}\left[\mathbf{R}^{\mathrm{I}}\right]+\min _{\rho^{\mathrm{I}}} E_{\mathrm{DFT}}\left[\rho^{\mathrm{I}}, \mathbf{R}^{\mathrm{I}}\right] .
$$

In this scheme, the forces on all atoms in region II are identical to forces on corresponding atoms if the classical potential were used for the entire system; i.e., the DFT region has no effect on these atoms. If the cutoff length of classical potential is $r_{c}$, then atoms that lie within region I and are farther than $r_{c}$ from the boundary will experience a force entirely from $E_{\mathrm{DFT}}[\mathrm{I}]$; these atoms feel a force no different than corresponding atoms in a DFT calculation of region I. The force on atoms in region I that are within $r_{c}$ of the boundary do not come entirely from $E_{\mathrm{DFT}}[\mathrm{I}]$, but also have contributions from $E_{\mathrm{cl}}[\mathrm{I}+\mathrm{II}]-E_{\mathrm{cl}}[\mathrm{I}]$. These contributions should in principle be corrections to the surface forces experienced by these atoms from $E_{\mathrm{DFT}}[\mathrm{I}]$. Classical potentials have been developed to mimic the energetics, forces, and geometries obtained from DFT calculations of various configurations, including surfaces; $;^{22}$ such potentials should be particularly apt for the present coupling scheme.

The implementation of this method demands nothing beyond what is required for a DFT calculation and a classical potential calculation. It should be noted, however, that the DFT calculation, $E_{\mathrm{DFT}}[\mathrm{I}]$, is a nonperiodic calculation, and if OF-DFT is to be used, special considerations may need to be made for the calculation of nonperiodic systems. $^{24}$

\section{B. Quantum interaction energy}

Alternatively $E^{\text {int }}$ can be calculated more accurately with a quantum-mechanical method. Although we only represent region II by the coordinates of the ions of region II atoms and calculate the energetics with a classical potential, there is an implicit charge density $\rho^{\mathrm{II}}$ associated with $E_{\mathrm{cl}}\left[\mathbf{R}^{\mathrm{II}}\right]$ via Eq. (8). Because of this, we can consider a more sophisticated coupling scheme where the interaction energy is based on density-functional theory. However, in order to compute the interaction energy via DFT when all we know about region II is an approximation of its charge density, the traditional Kohn-Sham scheme of DFT is not suitable. In the Kohn-Sham scheme, we start with a potential and obtain the density and energy of electrons in this potential. Instead, we need a means of calculating the energy of a system of electrons given their density. OF-DFT allows us to do this. Thus we can write down the interaction energy in terms of OF-DFT energy functionals:

$$
E^{\text {int }}[\mathrm{I}, \mathrm{II}]=E_{\mathrm{OF}}[\mathrm{I}+\mathrm{II}]-E_{\mathrm{OF}}[\mathrm{I}]-E_{\mathrm{OF}}[\mathrm{II}] .
$$

At first glance this seems like a useless scheme, because if DFT is used to calculate $E^{\text {int }}[\mathrm{I}, \mathrm{II}]$, we may as well use DFT to calculate $E[\mathrm{I}+\mathrm{II}]$, and thus no computational expense is saved with the coupled method. But because of the nature of many of the useful OF-DFT functionals, this turns out not to be the case. If $E^{\text {int }}[\mathrm{I}, \mathrm{II}]$ is calculated with OF-DFT, for typical approximate kinetic energy functionals the computation in Eq. (10) will require a computation time that is on the order of the computation time required to compute $E_{\mathrm{OF}}[\mathrm{I}]$, the small subsystem, rather than the time required to compute $E_{\mathrm{OF}}[\mathrm{I}+\mathrm{II}]$. This is because significant cancellation is implicit in $E_{\mathrm{OF}}[\mathrm{I}+\mathrm{II}]$ $-E_{\mathrm{OF}}[\mathrm{II}]$.

The existing approximate kinetic energy functionals differ in accuracy and computational efficiency. Moreover, differ- 
ent choices of functional can be made for the evaluation of $E[\mathrm{I}]$ and $E^{\text {int }}$, which further increases the number of possible coupling methods. This possibility is important because the degree to which the computation of $E^{\text {int }}$ can be made efficient depends on the choice of kinetic energy functional and the functionals that will most efficiently treat $E^{\text {int }}$ are not necessarily accurate enough to treat the interactions within $E[\mathrm{I}]$.

Regardless of the choice of kinetic energy functional, the evaluation of $E^{\text {int }}[\mathrm{I}, \mathrm{II}]$ within this coupling scheme requires knowing the electronic density of region II, $\rho^{\mathrm{II}}(\mathbf{r})$. In the present method, $\rho^{\mathrm{II}}(\mathbf{r})$ is approximated as the sum of atomic charge densities $\rho^{\text {at }}(\mathbf{r})$ centered at the region II nuclei:

$$
\rho^{\mathrm{II}}(\mathbf{r})=\sum_{i} \rho^{\mathrm{at}}\left(\mathbf{r}-\mathbf{R}_{i}^{\mathrm{II}}\right)
$$

This approximation is supported by the embedded-atom picture of simple metallic systems. In principle, $\rho^{\text {at }}(\mathbf{r})$ could be a nonspherically symmetric density. For example, if the arrangement of the region II atoms is always close to the bulk lattice arrangement, then a nonspherically symmetric charge density that reproduces the bulk charge density when periodically tiled may be more appropriate. However, in this article $\rho^{\text {at }}(\mathbf{r})$ is always taken to be spherically symmetric.

The density in region II is never explicitly represented in the calculation, but is given a precise form via Eq. (11). Thus region II is entirely described by the ionic coordinates $\mathbf{R}^{\mathrm{II}}$, and $\rho^{\mathrm{II}}$, the form of which is needed to evaluate $E^{\mathrm{int}}$, is implicitly determined by $\mathbf{R}^{\mathrm{II}}$.

The second coupling method is summarized by the expression for the energy as a function of nuclear coordinates within the method:

$$
\begin{aligned}
E\left[\mathbf{R}^{\mathrm{tot}}\right]= & E_{\mathrm{cl}}\left[\mathbf{R}^{\mathrm{II}}\right]+\underset{\rho^{I}}{\min }\left[E_{\mathrm{OF}}\left[\rho^{\mathrm{tot}} ; \mathbf{R}^{\mathrm{tot}}\right]-E_{\mathrm{OF}}\left[\rho^{\mathrm{II}} ; \mathbf{R}^{\mathrm{II}}\right]\right. \\
& \left.-E_{\mathrm{OF}}\left[\rho^{\mathrm{I}} ; \mathbf{R}^{\mathrm{I}}\right]+E_{\mathrm{DFT}}\left[\rho^{\mathrm{I}} ; \mathbf{R}^{\mathrm{I}}\right]\right]
\end{aligned}
$$

The last term, $E_{\mathrm{DFT}}\left[\rho^{\mathrm{I}} ; \mathbf{R}^{\mathrm{I}}\right]$, is written as such (and not as $\left.E_{\mathrm{OF}}[\mathrm{I}]\right)$ to emphasize that we could choose to compute it either with a Kohn-Sham-type calculation or with OF-DFT, but utilizing a more accurate kinetic energy functional than the other OF-DFT terms. This would allow for three distinct levels of accuracy in the calculation: Kohn-Sham accuracy within region I, OF-DFT accuracy for the coupling between regions I and II, and the accuracy of the classical potential in region II. In this case, $\rho^{\mathrm{I}}$ would consist of a set of KohnSham orbitals, $\rho^{\mathrm{I}}(\mathbf{r})=\sum_{i}\left|\psi_{i}(\mathbf{r})\right|^{2}$, and we would minimize over the $\psi_{i}$ :

$$
\begin{gathered}
E\left[\mathbf{R}^{\mathrm{tot}}\right]=E_{\mathrm{cl}}\left[\mathbf{R}^{\mathrm{II}}\right]+\min _{\psi_{i}}\left[E_{\mathrm{OF}}\left[\rho^{\mathrm{tot}} ; \mathbf{R}^{\mathrm{tot}}\right]-E_{\mathrm{OF}}\left[\rho^{\mathrm{II}} ; \mathbf{R}^{\mathrm{II}}\right]\right. \\
\left.-E_{\mathrm{OF}}\left[\rho^{\mathrm{I}} ; \mathbf{R}^{\mathrm{I}}\right]+E_{\mathrm{KS}}\left[\psi_{i} ; \mathbf{R}^{\mathrm{I}}\right]\right] \\
\rho^{\mathrm{tot}}=\sum_{i}\left|\psi_{i}\right|^{2}+\rho^{\mathrm{II}}
\end{gathered}
$$

$$
\rho^{\mathrm{I}}=\sum_{i}\left|\psi_{i}\right|^{2}
$$

However, this interesting possibility is not explored presently; instead we use the same type of OF-DFT calculation for the last four terms of Eq. (12). It should be noted that in this case the last two terms cancel, and then the total energy is given by

$$
E\left[\mathbf{R}^{\mathrm{tot}}\right]=E_{\mathrm{cl}}\left[\mathbf{R}^{\mathrm{II}}\right]+\underset{\rho^{I}}{\min }\left[E_{\mathrm{OF}}\left[\rho^{\mathrm{tot}} ; \mathbf{R}^{\mathrm{tot}}\right]-E_{\mathrm{OF}}\left[\rho^{\mathrm{II}} ; \mathbf{R}^{\mathrm{II}}\right]\right] .
$$

\section{Orbital-free DFT and approximate kinetic energy functionals}

Orbital-free DFT is a necessary part of the second coupling method, because the electronic structure of region II is represented only in terms of its density via Eq. (11); thus in order to utilize that information, $E^{\text {int }}$ must be based only on the charge density and the ionic coordinates. Here we describe some key ideas of OF-DFT.

Hohenberg and $\mathrm{Kohn}^{4}$ showed that the ground state energy of a system of electrons moving in an external potential is given by minimizing a density functional. Kohn and Sham $^{5}$ wrote a useful partitioning of this energy functional:

$$
E[\rho]=T_{s}[\rho]+E_{\mathrm{H}}[\rho]+E_{\mathrm{xc}}[\rho]+\int V_{\mathrm{e}-\mathrm{i}}(\mathbf{r}) \rho(\mathbf{r}) d \mathbf{r},
$$

where $T_{\mathrm{s}}$ is the noninteracting kinetic energy functional, $E_{\mathrm{H}}$ is the Hartree energy, $E_{\mathrm{xc}}$ is the exchange-correlation energy, and $V_{\mathrm{e}-\mathrm{i}}$ is the ionic potential. By introducing a set of fictitious noninteracting particles, we can obtain a set of singleparticle equations, the Kohn-Sham equations, that allow for the evaluation of $E[\rho]$ with an approximate $E_{\mathrm{xc}}$. The KohnSham method results in an exact evaluation of $T_{\mathrm{s}}\left[\rho_{0}\right]$ for the density $\rho_{0}$ that minimizes $E[\rho]$, but the method does not provide a means of evaluating $T_{s}[\rho]$ for an arbitrary density $\rho$.

The Kohn-Sham partitioning of the energy, Eq. (17), has turned out to be useful beyond the Kohn-Sham method. Because a number of limits of the exact noninteracting kinetic energy functional $T_{\mathrm{s}}[\rho]$ are known, ${ }^{25} T_{\mathrm{s}}[\rho]$ has been approximated by explicit density functionals constructed to satisfy one or more of these known limits. The orbital-free DFT methods are based on minimizing $E[\rho]$ with $T_{\mathrm{s}}$ replaced with an approximate kinetic energy functional.

OF-DFT methods are typically more computationally efficient than the Kohn-Sham method. If the approximate $T_{\mathrm{s}}$ can be evaluated with an amount of computation that scales linearly with the system size, usually denoted by the total number of atoms $N$, then minimizing $E_{\mathrm{OF}}[\rho]$ will require an amount of computation linear in the system size $[O(N)$ method]. Since within OF-DFT all terms of the energy are explicit functionals of the density, there is no need for fictitious orbitals, and the density $\rho(\mathbf{r})$ is the only represented 
variable. Thus, there is no need to solve the single-particle Scrhödinger equations for the fictitious particles while maintaining their orthogonality, operations that typically require most of the computational effort in the Kohn-Sham approach and scale as a high power of the system size $\left[O\left(N^{3}\right)\right.$ or higher]. Moreover, with the density $\rho(\mathbf{r})$ as the only quantity of interest in the system, the OF-DFT methods use less memory than the Kohn-Sham method, since the latter requires the storage and update of a number of fictitious orbitals proportional to the system size, each of which consumes twice the storage (as complex quantities) needed for the density alone. Orbital-free DFT methods can thus be used to study much larger systems than can be treated with orbital-based schemes, ${ }^{26}$ but with current computer capabilities are still limited to systems consisting of a few thousand atoms.

In addition to computational advantages, unlike the KohnSham method, the total energy functional $E_{\mathrm{OF}}[\rho]$ can be evaluated for a given $\rho(\mathbf{r})$. This property makes OF-DFT a suitable candidate for computing $E^{\text {int }}\left[\rho^{\mathrm{I}}, \rho^{\mathrm{II}}\right]$ in the second coupling method discussed in the preceding section.

The number of available approximate kinetic energy functionals is sizable, and the choice of functional is made based on considerations of efficiency and the types of systems to be treated. Because the systems to be considered are simple metals with free-electron-like charge densities, an important property that should be included in the approximate kinetic energy functional is the correct linear response around uniform densities:

$$
\hat{\mathcal{F}}\left[\left.\frac{\delta^{2} T_{\mathrm{s}}}{\delta \rho(\mathbf{r}) \delta \rho\left(\mathbf{r}^{\prime}\right)}\right|_{\rho 0}\right]=-\frac{1}{\chi_{\text {Lind }}(k)},
$$

where $\hat{\mathcal{F}}$ is the Fourier transform, and $\chi_{\text {Lind }}(k)$ is the Lindhard response function:

$$
\chi_{\text {Lind }}(k)=-\frac{k_{F}}{\pi^{2}}\left[\frac{1}{2}+\frac{1-x^{2}}{4 x} \ln \left|\frac{1+x}{1-x}\right|\right]
$$

with $k_{F}=\left(3 \pi^{2} \rho_{0}\right)^{1 / 3}$ and $x=k / 2 k_{F}$.

A significant number of efficient functionals have been developed that satisfy the linear response limit for a particular chosen average density. ${ }^{16-20,27}$ Such functionals often consist of several terms that are local or localized functionals, such as the Thomas-Fermi energy and the von Weizsäcker functionals, plus one or more convolution terms:

$$
T_{K}[\rho]=\int f(\rho(\mathbf{r})) K\left(\left|\mathbf{r}-\mathbf{r}^{\prime}\right|\right) g\left(\rho\left(\mathbf{r}^{\prime}\right)\right) d \mathbf{r} d \mathbf{r}^{\prime} .
$$

By choosing the kernel $K(r)$ properly, the approximate functional can be made to satisfy the correct linear response, Eq. (18), around some chosen uniform density $\rho_{0}$. Numerical tests indicate that among the current available efficient kinetic energy functionals, the ones of this form are most suitable for simple metallic systems. However, kinetic energy functionals that contain a convolution part with a long-range kernel make the efficient evaluation of $E^{\text {int }}[\mathrm{I}, \mathrm{II}]$ more computationally demanding; the consequences of this will be discussed in the following section. Although OF-DFT methods are more efficient than orbital-based DFT schemes, and have been successfully used to treat interesting physical systems (see, for example, Ref. 28), they are not as transferrable, and are better suited for metallic systems in which the electronic charge density does not deviate significantly from the uniform density limit.

\section{IMPLEMENTATION OF COUPLING}

\section{A. Classical interaction energy}

The calculation of the energetics and ionic forces within the first coupling scheme described above involve only open-boundary DFT calculations and classical potential calculations. As such, it is straightforward to use this method to perform multiscale simulations that combine the Kohn-Sham method for treating region I, and classical potentials for treating region II. We have successfully implemented this combination, but we leave the results of these calculations for a future presentation, since they treat a particular aspect of the coupling problem. Currently we focus on testing the merits of the multiscale methodology itself, and set aside the exploration of possible choices for the underlying energetic methods. Throughout this article OF-DFT is used to treat region I.

If the first coupling scheme is to be used for ionic relaxation, there are several possible numerical techniques, the optimal choice depending on the system being relaxed. If the partitioning of the system into regions I and II is such that the time required to calculate $E_{\mathrm{DFT}}[\mathrm{I}]$ is comparable with the computation time of $E_{\mathrm{cl}}[\mathrm{I}+\mathrm{II}]$, then ionic relaxation of the total system may be done by using a gradient-based minimizer such as conjugate gradient methods or quasiNewton methods like the Broyden-Fletcher-Goldfarb-Shanno (BFGS) method. ${ }^{29}$ If, on the other hand, the system partitioning is such that the time required to evaluate $E_{\mathrm{DFT}}[\mathrm{I}]$ is considerably more than that required for the computation of $E_{\mathrm{cl}}[\mathrm{I}+\mathrm{II}]$, as is often the case, then an alternate relaxation scheme may be more efficient. The total system can be relaxed by using a gradient-based minimizer on the region I system alone, while fully relaxing the region II ions between each ionic update of region I. Gradient-based minimizers like BFGS are only effective if the gradients involved are indeed gradients of an underlying object function. It is not immediately apparent that such is the case in this alternate-relaxation scheme, but we can demonstrate it as follows.

The energy calculated with the first coupling scheme, as a function of all ionic coordinates, is given in Eq. (9). A secondary function that only depends on the region I ionic positions can be defined as

$$
E^{\prime}\left[\mathbf{R}^{\mathrm{I}}\right] \equiv \min _{\mathbf{R}^{\mathrm{II}}} E\left[\mathbf{R}^{\mathrm{tot}}\right] .
$$

The useful aspect of $E^{\prime}$ is that its gradient with respect to $\mathbf{R}_{i}^{\mathrm{I}}$ can be easily evaluated:

$$
\frac{\partial E^{\prime}}{\partial \mathbf{R}_{i}^{\mathrm{I}}}=\frac{\partial E\left[\mathbf{R}^{\mathrm{tot}}\right]}{\partial \mathbf{R}_{i}^{\mathrm{I}}}+\sum_{j} \frac{\partial E\left[\mathbf{R}^{\mathrm{tot}}\right]}{\partial \mathbf{R}_{j}^{\mathrm{II}}} \frac{\partial \mathbf{R}_{j, \min }^{\mathrm{II}}}{\partial \mathbf{R}_{i}^{\mathrm{I}}}
$$




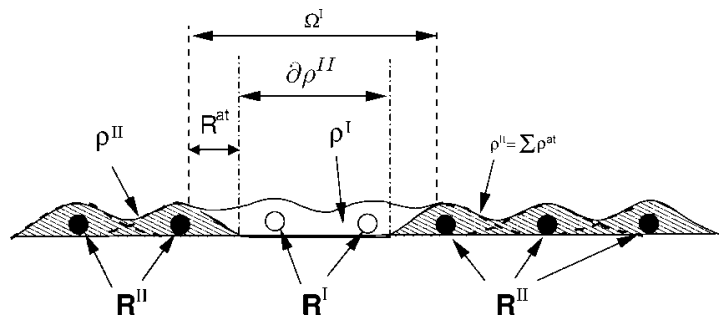

FIG. 1. An illustration of the partitioning of the system according to the coupling method with quantum interaction energy.

$$
=\frac{\partial E\left[\mathbf{R}^{\mathrm{tot}}\right]}{\partial \mathbf{R}_{i}^{\mathrm{I}}},
$$

where the second term on the right of Eq. (22) vanishes because all derivatives are evaluated at the minimum of $E\left[\mathbf{R}^{\text {tot }}\right]$ with respect to $\mathbf{R}^{\mathrm{II}}$. This result is analogous to the Hellmann-Feynman theorem. ${ }^{30}$ The introduction of the $E^{\prime}$ function allows for the following relaxation algorithm:

(i) Minimize $E\left[\mathbf{R}^{\text {tot }}\right]$ with respect to $\mathbf{R}^{\mathrm{II}}$ while holding $\mathbf{R}^{\mathrm{I}}$ fixed. This only involves the classical potential, $E_{\mathrm{cl}}\left[\mathbf{R}^{\mathrm{tot}}\right]$.

(ii) Calculate $\min _{\rho^{\mathrm{I}}} E_{\mathrm{DFT}}\left[\rho^{\mathrm{I}} ; \mathbf{R}^{\mathrm{I}}\right]$ and $E_{\mathrm{cl}}\left[\mathbf{R}^{\mathrm{I}}\right]$, and the forces on the region I ions. Using Eq. (23) the gradient of $E^{\prime}$ is obtained. $E^{\prime}$.

(iii) Perform a step of a gradient-based minimization of

(iv) Repeat until the system is relaxed.

In this manner, the number of DFT calculations performed is greatly reduced, albeit at the expense of more classical potential calculations.

\section{B. Quantum interaction energy}

Implementation details of the second coupling method require more elaboration. One important point is that $\rho^{\mathrm{I}}$ must be confined to lie within a finite volume $\Omega^{\mathrm{I}}$. This region should have significant overlap with the region where $\rho^{\mathrm{II}}$ lies, in order to provide coupling of the two regions. But if $\rho^{\mathrm{I}}$ were not confined to a finite volume $\Omega^{\mathrm{I}}$, it could in principle spread throughout the combined system, and during the course of minimizing with respect to $\rho^{\mathrm{I}}$ [Eq. (12)], we would essentially be performing a DFT calculation of the whole system. On the other hand, $\Omega^{\mathrm{I}}$ should be chosen large enough so that $\rho^{\mathrm{I}}$ is not artificially confined. In the test systems we examined, we found that when increasing the size of $\Omega^{\mathrm{I}}$, a point is reached where the results (e.g., the shape of $\rho^{\mathrm{I}}$ and the forces on the ions) change little. We found that a useful rule for determining the optimal size of $\Omega^{\mathrm{I}}$ is the following: Define $\partial \rho^{\mathrm{II}}$ to be the surface on which $\rho^{\mathrm{II}}=0$. Let the range of the atomic densities $\rho^{\text {at }}$ be $R^{\text {at }}$. $\Omega^{\mathrm{I}}$ should be chosen large enough so that for any point $\mathbf{r}$ within $\partial \rho^{\mathrm{II}}$, and any point $\mathbf{r}^{\prime}$ outside of $\Omega^{\mathrm{I}}$, we have $\left|\mathbf{r}-\mathbf{r}^{\prime}\right|>R^{\text {at }}$. The confinement of $\rho^{\mathrm{I}}$ within $\Omega^{\mathrm{I}}$, and the choice of $\Omega^{\mathrm{I}}$, is illustrated in Fig. 1.

The second coupling method maintains efficiency due to the cancellation that occurs when $E_{\mathrm{OF}}^{\mathrm{int}}$ is computed.
Consider the computation of a local term of $E^{\text {int }}$, such as the exchange-correlation functional within the local density approximation $^{4}$ (LDA):

$$
\begin{aligned}
E_{\mathrm{xc}}^{\mathrm{int}} & =\int f_{\mathrm{xc}}\left(\rho^{\mathrm{tot}}\right) d \mathbf{r}-\int_{\Omega^{I}} f_{\mathrm{xc}}\left(\rho^{\mathrm{I}}\right) d \mathbf{r}-\int f_{\mathrm{xc}}\left(\rho^{\mathrm{II}}\right) d \mathbf{r} \\
& =\int_{\Omega^{\mathrm{I}}}\left[f_{\mathrm{xc}}\left(\rho^{\mathrm{tot}}\right)-f_{\mathrm{xc}}\left(\rho^{\mathrm{I}}\right)-f_{\mathrm{xc}}\left(\rho^{\mathrm{II}}\right)\right] d \mathbf{r},
\end{aligned}
$$

where $f_{\mathrm{xc}}(\rho) \equiv \rho \epsilon_{\mathrm{xc}}(\rho)$ and we have used the fact that $\rho^{\mathrm{II}}(\mathbf{r})=\rho^{\mathrm{tot}}(\mathbf{r})$ for $\mathbf{r} \notin \Omega^{\mathrm{I}}$. Thus calculation of $E_{\mathrm{xc}}^{\mathrm{int}}$ is an integral over $\Omega^{\mathrm{I}}$ and not the entire system, which demonstrates our criterion for efficiency. Any local functional of $\rho$ will obviously be calculated efficiently in the same manner. We note that when the same kinetic energy functional is used for the interaction energy and $E_{\mathrm{OF}}[\mathrm{I}]$ (which is the case for the tests performed in this paper), the cancellation exhibited in Eq. (16) occurs. In this case, it is wasteful to compute the interaction energy as in Eq. (24) and then compute and add on $E_{\mathrm{xc}}\left[\rho^{\mathrm{I}}\right]$, as it exactly cancels the second term of Eq. (24). Instead, we compute directly the following quantity:

$$
E_{\mathrm{xc}}^{\mathrm{int}+\mathrm{I}}=\int_{\Omega^{\mathrm{I}}}\left[f_{\mathrm{xc}}\left(\rho^{\mathrm{tot}}\right)-f_{\mathrm{xc}}\left(\rho^{\mathrm{II}}\right)\right] d \mathbf{r} .
$$

Similar considerations apply to the other parts of the energy that are simple functionals of the density. The only term that does not fall in this category is the interaction kinetic energy $T_{\mathrm{s}}^{\mathrm{int}}$, when it involves more sophisticated functionals with convolution terms such as Eq. (20). For this case, we have developed an appropriate efficient methodology, the derivation of which is contained in the Appendix.

Particular attention must be paid to the nonlocal terms of $E^{\text {int }}$. As usual, cancellation occurs among electron-electron, electron-ion, and ion-ion terms, which eliminates longranged interactions. The Hartree interaction energy is given by

$$
\begin{aligned}
E_{\mathrm{H}}^{\mathrm{int}}= & \frac{1}{2} \int \frac{\rho^{\mathrm{tot}}(\mathbf{r}) \rho^{\mathrm{tot}}\left(\mathbf{r}^{\prime}\right)}{\left|\mathbf{r}-\mathbf{r}^{\prime}\right|} d \mathbf{r} d \mathbf{r}^{\prime} \\
& -\frac{1}{2} \int \frac{\rho^{\mathrm{I}}(\mathbf{r}) \rho^{\mathrm{I}}\left(\mathbf{r}^{\prime}\right)+\rho^{\mathrm{II}}(\mathbf{r}) \rho^{\mathrm{II}}\left(\mathbf{r}^{\prime}\right)}{\left|\mathbf{r}-\mathbf{r}^{\prime}\right|} \mathrm{d} \mathbf{r} d \mathbf{r}^{\prime} \\
= & \int \frac{\rho^{\mathrm{I}}(\mathbf{r}) \rho^{\mathrm{II}}\left(\mathbf{r}^{\prime}\right)}{\left|\mathbf{r}-\mathbf{r}^{\prime}\right|} d \mathbf{r} d \mathbf{r}^{\prime} \\
= & \int_{\Omega^{\mathrm{I}}} \rho^{\mathrm{I}}(\mathbf{r}) \sum_{i} V_{\mathrm{H}}^{\mathrm{at}}\left(\mathbf{r}-\mathbf{R}_{i}^{\mathrm{II}}\right) d \mathbf{r},
\end{aligned}
$$

where

$$
V_{\mathrm{H}}^{\mathrm{at}}(\mathbf{r}) \equiv \int \frac{\rho^{\mathrm{at}}\left(\mathbf{r}^{\prime}\right)}{\left|\mathbf{r}-\mathbf{r}^{\prime}\right|} d \mathbf{r}^{\prime}
$$

Similarly the electron-ion interaction energy $E_{e-i}^{\text {int }}$ reduces to 


$$
\begin{aligned}
E_{e-i}^{\mathrm{int}}= & \int_{\Omega^{I}} \rho^{\mathrm{I}}(\mathbf{r}) \sum_{i} V_{\mathrm{psp}}\left(\mathbf{r}-\mathbf{R}_{i}^{\mathrm{II}}\right) d \mathbf{r} \\
& +\int \sum_{j} \rho^{\mathrm{at}}\left(\mathbf{r}-\mathbf{R}_{j}^{\mathrm{II}}\right) \sum_{i} V_{\mathrm{psp}}\left(\mathbf{r}-\mathbf{R}_{i}^{\mathrm{I}}\right) d \mathbf{r},
\end{aligned}
$$

where $V_{\mathrm{psp}}(r)$ is the pseudopotential representing the ion, and we have used Eq. (11) to express $\rho^{\mathrm{II}}(\mathbf{r})$ as a sum of $\rho^{\text {at }}$. Finally the ion-ion interaction energy is given by

$$
E_{i-i}^{\mathrm{int}}=\sum_{i, j} \frac{Z_{i} Z_{j}}{\left|\mathbf{R}_{i}^{\mathrm{I}}-\mathbf{R}_{j}^{\mathrm{II}}\right|}
$$

The combination of all three Coulomb terms can be expressed as

$$
\begin{aligned}
E_{\mathrm{H}}^{\mathrm{int}}+E_{e-i}^{\mathrm{int}}+E_{i-i}^{\mathrm{int}}= & \int_{\Omega^{\mathrm{I}}} \rho^{\mathrm{I}}(\mathbf{r})\left[\sum_{i} V_{\mathrm{elec}}^{\mathrm{at}}\left(\mathbf{r}-\mathbf{R}_{i}^{\mathrm{II}}\right)\right] d \mathbf{r} \\
& +\sum_{i, j} \phi_{i j}\left(\mathbf{R}_{i}^{\mathrm{I}}-\mathbf{R}_{j}^{\mathrm{II}}\right),
\end{aligned}
$$

where we have defined

$$
\begin{gathered}
V_{\mathrm{elec}}^{\mathrm{at}}(\mathbf{r}) \equiv V_{\mathrm{H}}^{\mathrm{at}}(\mathbf{r})+V_{\mathrm{psp}}(\mathbf{r}), \\
\phi_{i j}\left(\mathbf{R}_{i}^{\mathrm{I}}-\mathbf{R}_{j}^{\mathrm{II}}\right) \equiv \frac{Z_{i} Z_{j}}{\left|\mathbf{R}_{i}^{\mathrm{I}}-\mathbf{R}_{j}^{\mathrm{II}}\right|}+\int V_{\mathrm{psp}}\left(\mathbf{r}-\mathbf{R}_{i}^{\mathrm{I}}\right) \rho^{\mathrm{at}}\left(\mathbf{r}-\mathbf{R}_{j}^{\mathrm{II}}\right) d \mathbf{r} .
\end{gathered}
$$

Both $V_{\text {elec }}^{\text {at }}(\mathbf{r})$ and $\phi_{i j}(\mathbf{R})$ are short-ranged functions in which the $1 / R$ dependence of the constituent terms cancel.

Within the second coupling method: (1) we minimize the energy with respect to $\rho^{I}$, and (2) we calculate the forces on all of the ions and update their position. In order to minimize the energy with respect to $\rho^{\mathrm{I}}$, the derivative $\delta E^{\text {int }} / \delta \rho^{\mathrm{I}}(\mathbf{r})$ needs to be calculated for $\mathbf{r} \in \Omega^{I}$. This derivative can be evaluated efficiently for the local functionals like $E_{\text {xc }}$ :

$$
\frac{\delta E_{\mathrm{xc}}^{\mathrm{int}}}{\delta \rho^{\mathrm{I}}(\mathbf{r})}=f_{\mathrm{xc}}^{\prime}\left(\rho^{\mathrm{tot}}\right)-f_{\mathrm{xc}}^{\prime}\left(\rho^{\mathrm{I}}\right)
$$

where $f_{\mathrm{xc}}^{\prime}=d f_{\mathrm{xc}} / d \rho$. For the long-ranged Coulombic functionals, the derivative is given by

$$
\frac{\delta}{\delta \rho^{\mathrm{I}}(\mathbf{r})}\left[E_{\mathrm{H}}^{\mathrm{int}}+E_{e-i}^{\mathrm{int}}+E_{i-i}^{\mathrm{int}}\right]=\sum_{i} V_{\mathrm{elec}}^{\mathrm{at}}\left(\mathbf{r}-\mathbf{R}_{i}^{\mathrm{II}}\right)
$$

Evaluating this combined contribution to $\delta E^{\mathrm{int}} / \delta \rho^{\mathrm{I}}$ is a simple matter of evaluating $V_{\text {elec }}^{\text {at }}$ for region II ions located near the boundary with region I. And so the gradient of the total energy with respect to $\rho^{\mathrm{I}}$ is

$$
\frac{\delta E}{\delta \rho^{\mathrm{I}}(\mathbf{r})}=\frac{\delta E_{\mathrm{DFT}}\left[\rho^{I} ; \mathbf{R}^{\mathrm{I}}\right]}{\delta \rho^{\mathrm{I}}(\mathbf{r})}+\frac{\delta E_{\mathrm{xc}}^{\mathrm{int}}}{\delta \rho^{\mathrm{I}}(\mathbf{r})}+\frac{\delta T_{s}^{\mathrm{int}}}{\delta \rho^{\mathrm{I}}(\mathbf{r})}+\sum_{i} V_{\mathrm{elec}}^{\mathrm{at}}\left(\mathbf{r}-\mathbf{R}_{i}^{\mathrm{II}}\right)
$$

The calculation of the ionic forces proceeds differently for region I and region II ions. Calculation of the region I ionic forces is facilitated by the Hellmann-Feynman theorem. ${ }^{30}$ If we denote the part of the energy [Eq. (12)] that is minimized with respect to $\rho^{\mathrm{I}}$ by $G\left[\rho^{\mathrm{I}} ; \mathbf{R}^{\mathrm{I}}, \mathbf{R}^{\mathrm{II}}\right]$ :

$$
G\left[\rho^{\mathrm{I}} ; \mathbf{R}^{\mathrm{I}}, \mathbf{R}^{\mathrm{II}}\right] \equiv E_{\mathrm{OF}}[\mathrm{I}+\mathrm{II}]-E_{\mathrm{OF}}[\mathrm{II}]-E_{\mathrm{OF}}[\mathrm{I}]+E_{\mathrm{DFT}}[\mathrm{I}],
$$

then we have, for the second coupling scheme:

$$
E\left[\mathbf{R}^{\mathrm{tot}}\right]=E^{\mathrm{cl}}\left[\mathbf{R}^{\mathrm{II}}\right]+\min _{\rho^{\mathrm{I}}} G\left[\rho^{\mathrm{I}} ; \mathbf{R}^{\mathrm{I}}, \mathbf{R}^{\mathrm{II}}\right],
$$

and when forces on region I ions are computed, the expression simplifies:

$$
\frac{\partial E\left[\mathbf{R}^{\mathrm{tot}}\right]}{\partial \mathbf{R}_{i}^{\mathrm{I}}}=\frac{\partial G}{\partial \mathbf{R}_{i}^{\mathrm{I}}}+\int_{\Omega^{\mathrm{I}}} \frac{\delta G}{\delta \rho^{\mathrm{I}}(\mathbf{r})} \frac{\partial \rho_{\min }^{\mathrm{I}}(\mathbf{r})}{\partial \mathbf{R}_{i}^{\mathrm{I}}} d \mathbf{r}
$$

$$
=\frac{\partial G}{\partial \mathbf{R}_{i}^{\mathrm{I}}}
$$

where the last term in Eq. (37) vanishes because we have minimized $G$ with respect to $\rho^{\mathrm{I}}$, and so $\delta G /\left.\delta \rho^{\mathrm{I}}\right|_{\rho_{\min }^{\mathrm{I}}}=0$. So the forces on the region I ions are determined solely by the terms of $G$ that explicitly depend on $\mathbf{R}^{\mathrm{I}}$; these terms are the electron-ion energy and the ion-ion energy. Using Eq. (30), the force on the $i$ th region I ion is given by

$$
\begin{aligned}
-\mathbf{F}_{i}^{\mathrm{I}} & =\frac{\partial E\left[\mathbf{R}^{\mathrm{tot}}\right]}{\partial \mathbf{R}_{i}^{\mathrm{I}}}=\frac{\partial}{\partial \mathbf{R}_{i}^{\mathrm{I}}}\left(E_{\mathrm{e}-\mathrm{i}}[\mathrm{I}]+E_{\mathrm{i}-\mathrm{i}}[\mathrm{I}]+E_{\mathrm{e}-\mathrm{i}}^{\mathrm{int}}[\mathrm{I}, \mathrm{II}]+E_{\mathrm{i}-\mathrm{i}}^{\mathrm{int}}[\mathrm{I}, \mathrm{II}]\right) \\
& =\frac{\partial}{\partial \mathbf{R}_{i}^{\mathrm{I}}}\left(E_{\mathrm{e}-\mathrm{i}}[\mathrm{I}]+E_{\mathrm{i}-\mathrm{i}}[\mathrm{I}]\right)+\sum_{j} \boldsymbol{\nabla} \phi_{i j}\left(\mathbf{R}_{i}^{\mathrm{I}}-\mathbf{R}_{j}^{\mathrm{II}}\right) .
\end{aligned}
$$

Thus it can be seen from Eq. (39) that forces on region I ions are given by the sum of the electron-ion and ion-ion forces present in subsystem I alone, and short-ranged interactions with region II ions that are nearby region $\mathrm{I}$.

The forces on the region II ions come mostly from the classical potential, but they have contributions from $E^{\text {int }}[\mathrm{I}, \mathrm{II}]$ because $\rho^{\mathrm{II}}$ is a function of $\mathbf{R}_{j}^{\mathrm{II}}$. Since we have not minimized with respect to $\rho^{\mathrm{II}}$, there is no Hellmann-Feynman simplification when calculating the forces on region II ions, and all terms in the interaction energy contribute. The force on the $j$ th region II ion is given by

$$
-\mathbf{F}_{j}^{\mathrm{II}}=\frac{\partial E\left[\mathbf{R}^{\mathrm{tot}}\right]}{\partial \mathbf{R}_{j}^{\mathrm{II}}}=\frac{\partial E^{\mathrm{cl}}\left[\mathbf{R}^{\mathrm{II}}\right]}{\partial \mathbf{R}_{j}^{\mathrm{II}}}+\frac{\partial E^{\mathrm{int}}[\mathrm{I}, \mathrm{II}]}{\partial \mathbf{R}_{j}^{\mathrm{II}}} .
$$

Local functional parts of $E^{\text {int }}$ such as the exchangecorrelation energy will have a contribution to the force given by 


$$
\frac{\partial E_{\mathrm{xc}}^{\mathrm{int}}}{\partial \mathbf{R}_{j}^{\mathrm{II}}}=-\int_{\Omega^{I}} \boldsymbol{\nabla} \rho^{\mathrm{at}}\left(\mathbf{r}-\mathbf{R}_{j}^{\mathrm{II}}\right)\left[f_{\mathrm{xc}}^{\prime}\left(\rho^{\mathrm{tot}}\right)-f_{\mathrm{xc}}^{\prime}\left(\rho^{\mathrm{II}}\right)\right] d \mathbf{r}
$$

with analogous expressions for other local contributions that may exist in the kinetic energy functional such as the Thomas-Fermi energy. These local force contributions are only non-zero for region II ions with an atomic density that extends into $\Omega^{\mathrm{I}}$. It is also worth noting that the integral in Eq. (41) need not be carried out over all of $\Omega^{\mathrm{I}}$, but only over the intersection of $\Omega^{\mathrm{I}}$ with $\rho^{\text {at }}\left(\mathbf{r}-\mathbf{R}_{j}^{\mathrm{II}}\right)$.

The Coulomb contributions to the region II ionic forces are given by

$$
\begin{aligned}
\frac{\partial}{\partial \mathbf{R}_{j}^{\mathrm{II}}}\left[E_{\mathrm{e}-\mathrm{i}}^{\mathrm{int}}+E_{\mathrm{i}-\mathrm{i}}^{\mathrm{int}}+E_{\mathrm{H}}^{\mathrm{int}}\right]= & -\sum_{i} \boldsymbol{\nabla} \phi_{i j}\left(\mathbf{R}_{i}^{\mathrm{I}}-\mathbf{R}_{j}^{\mathrm{II}}\right) \\
& -\int_{\Omega^{\mathrm{I}}} \rho^{\mathrm{I}}(\mathbf{r}) \boldsymbol{\nabla} V_{\mathrm{elec}}^{\mathrm{at}}\left(\mathbf{r}-\mathbf{R}_{j}^{\mathrm{II}}\right) d \mathbf{r} .
\end{aligned}
$$

This contribution also is nonzero only for region II ions near the boundary of $\Omega^{\mathrm{I}}$.

If a more sophisticated kinetic energy functional with a convolution term such as Eq. (20) is used in $E^{\text {int }}$, then such a term adds considerable complication to the calculation of the forces on region II ions, but these contributions nonetheless die off as we move farther from region I. Thus within the framework of this coupling scheme, the forces on region II ions take the intuitively satisfying form of being equal to the force that arises from the classical potential, plus a correction force for ions near the boundary of $\Omega^{\mathrm{I}}$.

If the partitioning of the system between parts I and II is such that region I requires a much longer computation than region II, the second coupling method, like the first, allows for an efficient algorithm for ionic relaxation. We define a different partitioning of the ions as follows: we denote by $\mathbf{R}^{\mathrm{I}^{\prime}}$ the set of region I ions plus all region II ions $\mathbf{R}_{j}^{\mathrm{II}}$ for which the interaction force $\partial E^{\text {int }} / \partial \mathbf{R}_{j}^{\mathrm{II}}$ is not negligible, and we denote by $\mathbf{R}^{\mathrm{II}^{\prime}}$ the rest of the $\mathbf{R}^{\mathrm{II}}$ ions. The point is that the forces on the $\mathbf{R}^{\text {II' }}$ ions only depend on the classical potential [as seen from Eq. (40)], and also that $\rho^{\mathrm{I}}$ does not depend on the $\mathbf{R}^{\mathrm{II}}$ ions [as seen from Eq. (34)]. Thus the same algorithm for relaxing the system in the first coupling scheme can be used, but with $\mathbf{R}^{\mathrm{I}}$ replaced with $\mathbf{R}^{\mathrm{I}^{\prime}}$, and $\mathbf{R}^{\mathrm{II}}$ replaced with $\mathbf{R}^{\mathrm{II}^{\prime}}$. That is, before each relaxation step of the $\mathbf{R}^{\mathrm{I}^{\prime}}$, the $\mathbf{R}^{\mathrm{II}^{\prime}}$ are to be fully relaxed.

\section{TESTS}

In order to test the present coupling methods, we have focused on a simple coupled system that is readily analyzed. The system consists of $10 \times 10 \times 10$ cubic unit cells $(4$ atoms each) of crystalline fcc aluminum. The innermost $2 \times 2 \times 2$ unit cells (32 atoms total) are considered to be region I, and all atoms outside are considered to be in region II. Region II, which is treated with the classical potential, is treated as a periodic system in order to remove effects of surfaces from the simulation. So in fact the test system consists of an infi-

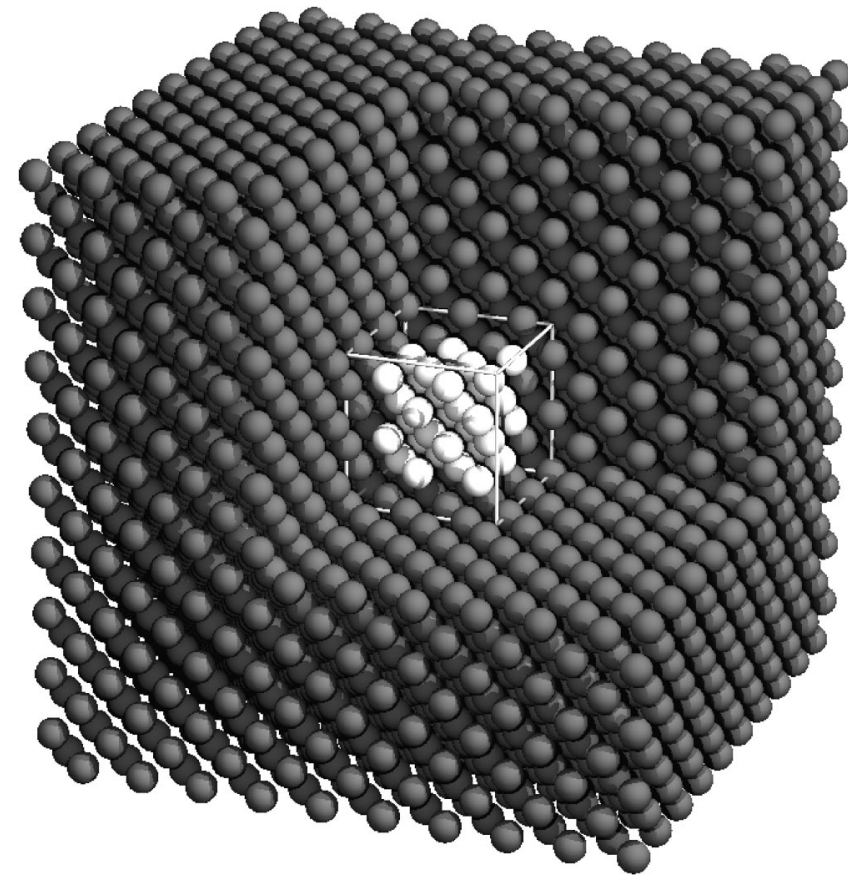

FIG. 2. A cutaway view of the test system. White atoms belong to region I, and dark atoms belong to region II. For the coupling method with quantum interaction energy, the region $\Omega^{\mathrm{I}}$ is shown by the white cube.

nite array of 32-atom $\mathrm{Al}$ clusters treated quantum mechanically, embedded in an Al bulk treated by classical potentials. Obviously, if there is good coupling between region I, and region II, the entire system should simply behave like pure bulk fcc Al, making it easy to evaluate the quality of the coupling. This test system is illustrated in Fig. 2. Additionally, we have applied the quantum interaction coupling method to a more interesting physical system, a screw dislocation in aluminum.

In all of the present tests, region I is treated with OF-DFT. However, the particular kinetic energy functional used differs among the tests. The $\mathrm{Al}$ ions are represented with the Goodwin-Needs-Heine local pseudopotential. ${ }^{31}$ For all tests, the classical potential used is the "glue" potential of Ercolessi and co-workers, ${ }^{12}$ which has the embedded-atom method (EAM) form:

$$
E\left[\mathbf{R}_{i}\right]=\sum_{i} F\left[\sum_{i \neq j} \rho^{\operatorname{EAM}}\left(\left|\mathbf{R}_{i j}\right|\right)\right]+\frac{1}{2} \sum_{i \neq j} \phi\left(\left|\mathbf{R}_{i j}\right|\right) .
$$

The EAM potential has been scaled both in $\mathbf{r}$ and in energy:

$$
\begin{gathered}
F[\rho] \rightarrow \alpha F[\rho], \\
\rho^{\mathrm{EAM}}(\mathbf{R}) \rightarrow \rho^{\mathrm{EAM}}(\beta \mathbf{R}), \\
\phi(\mathbf{R}) \rightarrow \alpha \phi(\beta \mathbf{R})
\end{gathered}
$$

with $\alpha$ and $\beta$ chosen so that the potential yields precisely the same lattice constant and bulk modulus of fcc Al simulated with OF-DFT employing the particular kinetic energy functional used in that test. This is in accord with the 
philosophy that the coupled simulation should behave as if the accurate method were used for the entire system. But this procedure is also done so that a "fair" assessment of the coupling itself can be made; we wish to examine errors in the present coupling methods themselves and the approximations involved in them, but not the errors coming from a trivial incompatibility between energy methods. To make the classical potential even more compatible with the OF-DFT method, we could redetermine the form of the classical potential using the method that Ercolessi and Adams originally used to develop their potential: ${ }^{22}$ perform a large number of reference energetic calculations of $\mathrm{Al}$ using OF-DFT, and find the EAM-type potential that best reproduces these results. This would be a rather involved procedure, so we have chosen to simply scale the potential.

\section{A. Test of classical interaction energy method}

In the first coupling method [Eq. (9)], the energetics of region I was treated with OF-DFT, and the kinetic energy functional used was one developed by Wang et al. ${ }^{20}$ with a density-dependent kernel and parameters $\left\{\alpha, \beta, \gamma, \rho_{*}\right\}=\left\{5 / 6+\sqrt{5} / 6,5 / 6-\sqrt{5} / 6,2.7,0.183 \AA^{-3}\right\}$ (in the notation of Ref. 20). This functional has six convolution terms of the form of Eq. (20). The classical potential was scaled to match the lattice constant $(4.033 \AA)$ and bulk modulus $(55.7 \mathrm{GPa})$ of fcc $\mathrm{Al}$ obtained by this kinetic energy functional.

The system was initially arranged in the perfect fcc lattice configuration. The forces on the region II atoms were identically zero, since they come entirely from $E^{\mathrm{cl}}[\mathrm{I}+\mathrm{II}]$, which is at a minimum in the initial configuration. However, the forces on the region I atoms are not zero, as the OF-DFT and EAM forces do not perfectly cancel each other. The average magnitude of the forces on the region I atoms was $0.33 \mathrm{eV} / \AA$, and the maximum force on a region I atom was $0.45 \mathrm{eV} / \mathrm{A}$. These initial forces on the region I atoms are shown in Fig. 3(a), with the drawn force vectors scaled so that a force of $1 \mathrm{eV} / \AA$ would extend one lattice constant. Then the coupled system was relaxed. After relaxation, it was found that the atomic positions deviated from the correct fcc lattice positions by an average (over all atoms in the system) of $0.004 \AA$ per atom. The average deviation of just the region I atoms was $0.07 \AA$ per atom. The atomic deviation is shown in Fig. 3(b), in which the relaxed atomic positions for the region I and region II atoms are drawn as white and black balls, respectively, and the correct lattice positions are drawn as gray balls of a slightly smaller radius. Note that only the four (100) layers that include region I are shown. From this diagram it can be seen that in general the relaxed atomic positions deviate from the the perfect lattice positions by bulging out from region I slightly, with the deviation decreasing with increasing distance from region I.

\section{B. Test of quantum interaction energy method}

The second coupling method was applied to the same simple test system. The kinetic energy functional (a)
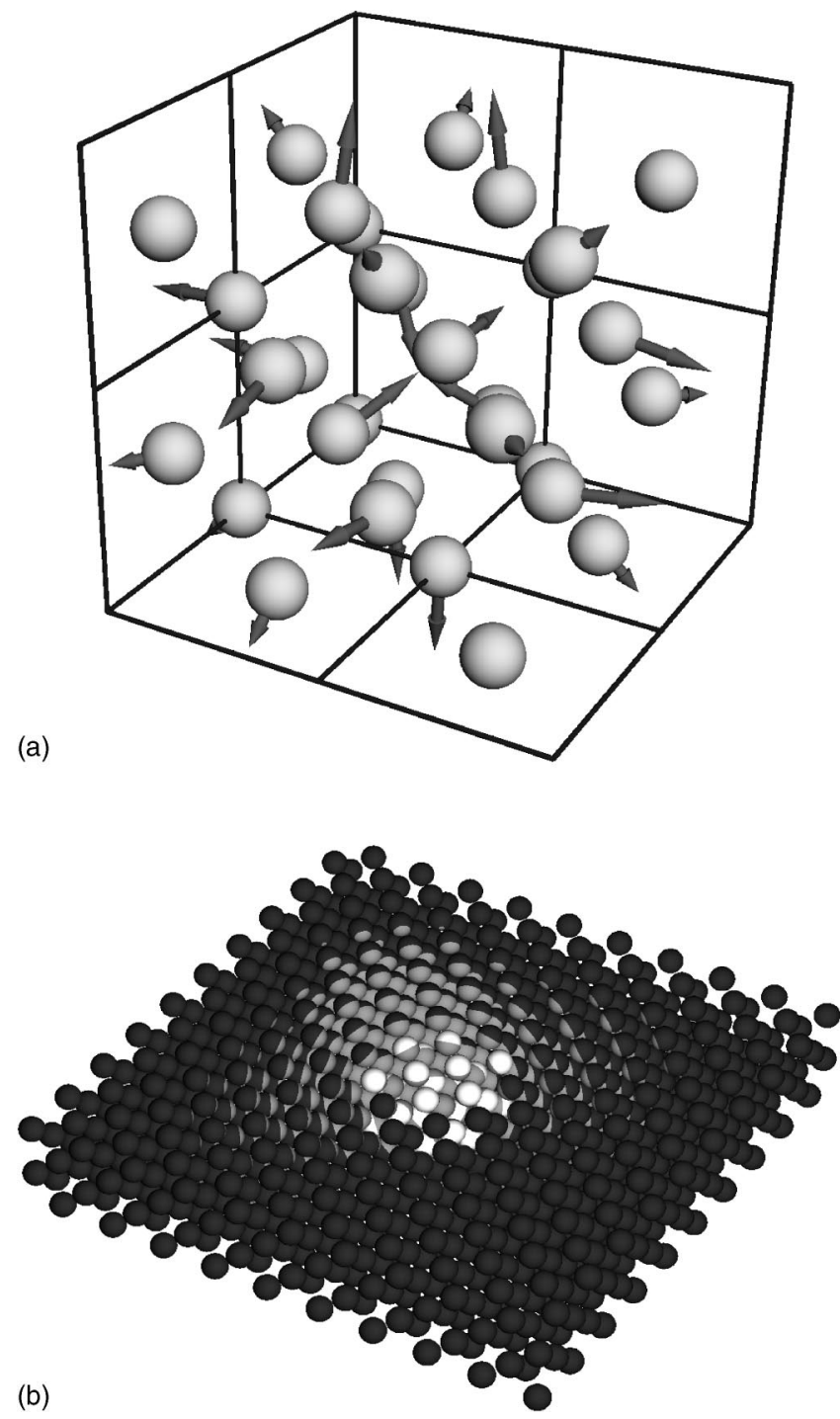

FIG. 3. Test of the coupling method with classical interaction energy. (a) The forces on region I atoms when the atomic positions are at the perfect lattice positions. The force factors are scaled so that a vector of length one lattice constant corresponds to $1 \mathrm{eV} / \AA$. (b) The relaxed region I and two atomic positions shown in white and black, respectively. The perfect lattice sites are drawn as gray spheres of a slightly smaller radius.

employed was again from Ref. 20, but in this case it was a functional with a density-independent kernel, with parameters $\{\alpha, \beta\}=\{5 / 6 \pm \sqrt{5} / 6\}$. A different functional was chosen for this test because of its simpler form: it contains only one convolution term of the form of Eq. (20), while the functional used in the test of the first coupling method had six. This makes the evaluation of the kinetic interaction energy, $T_{\mathrm{s}}^{\text {int }}$, simpler. Furthermore, this functional performs well for structures that do not deviate much from the bulk system. This functional was found to be inapplicable to the test of the first coupling method, because in that approach the calculation of $E_{\mathrm{OF}}[\mathrm{I}]$ amounts to an isolated cluster; in that case, there is no embedding potential from region II and the minimization with respect to $\rho^{\mathrm{I}}$ does not converge. For bulk fcc $\mathrm{Al}$, however, this sim- 


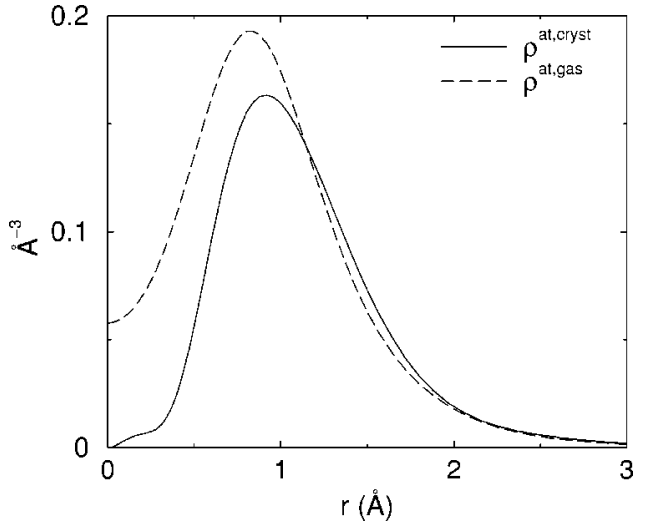

FIG. 4. The two atomic densities used to represent $\rho^{\mathrm{II}}$.

pler functional employed to test the quantum interaction energy method performs quite well, producing an equilibrium lattice constant of $4.035 \AA$ and a bulk modulus of $71.9 \mathrm{GPa}$.

Another aspect of the second coupling method is the choice of atomic density functions $\rho^{\text {at }}$ representing $\rho^{\mathrm{II}}$ through Eq. (11). Two different choices of $\rho^{\text {at }}$ were tried. One choice, $\rho^{\text {at,gas }}$, was the valence density from a Kohn-Sham calculation of an isolated $\mathrm{Al}$ atom, represented with the same

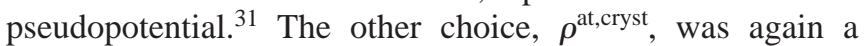
spherically symmetric charge density chosen such that the charge density that results from periodically superposing it on an fcc lattice most closely matches the charge density coming from an OF-DFT calculation of bulk fcc Al. The desired spherically symmetric charge density $\rho^{\text {at,cryst }}(r)$ minimizes:

$$
\int_{\Omega}\left[\left(\delta^{\mathrm{fcc} *} \rho^{\mathrm{at}, \mathrm{cryst}}\right)(\mathbf{r})-\rho^{\mathrm{fcc}}(\mathbf{r})\right]^{2} d \mathbf{r},
$$

where $\delta^{\mathrm{fcc}}(\mathbf{r})$ is an infinite fcc lattice of $\delta$ functions, * denotes convolution, and $\Omega$ is one unit cell; $\rho^{\mathrm{fcc}}(\mathbf{r})$ is the valence charge density of the fcc crystal. In reciprocal space, this becomes

$$
\Omega \sum_{\mathbf{Q}}\left|\widetilde{S}_{\mathbf{Q}} \widetilde{\rho}^{\mathrm{at}, \mathrm{cryst}}(Q)-\widetilde{\rho}_{\mathbf{Q}}^{\mathrm{fcc}}\right|^{2}
$$

where $\tilde{S}_{\mathbf{Q}}$ is the structure factor. This is minimized by requiring

$$
\tilde{\rho}^{\mathrm{at}, \mathrm{cryst}}(Q)=\frac{\left\langle\tilde{\rho}_{\mathbf{Q}}^{\mathrm{fcc}}\right\rangle_{Q}}{\left\langle\widetilde{S}_{\mathbf{Q}}\right\rangle_{Q}},
$$

where $\langle\cdots\rangle_{Q}$ denotes an averaging over reciprocal lattice vectors $\mathbf{Q}$ of length $Q$. This $\widetilde{\rho}^{\text {at,cryst }}(Q)$ was then used to construct a radial charge density $\rho^{\text {at,cryst }}(r)$ that was commensurate with $\widetilde{\rho}^{\text {at,cryst }}(Q)$ at the values of $Q$ where the latter was defined. The two charge density choices are shown in Fig. 4.

The second coupling method was tested on the same system used to test the first coupling method. With the second method, however, we must choose the form of the atomic density representing $\rho^{\mathrm{II}}$ and the extent of the region $\Omega^{\mathrm{I}}$. With respect to the choice of $\Omega^{\mathrm{I}}$, we have found the following general behavior: if $\Omega^{\mathrm{I}}$ is chosen to be too small, then after minimization with respect to $\rho^{\mathrm{I}}$, there is an excess buildup of charge near the boundary of $\Omega^{\mathrm{I}}$. This in turn results in a net attraction of the region $\mathrm{I}$ ions toward the boundary of $\Omega^{\mathrm{I}}$. This is remedied by an increase in the size of $\Omega^{\mathrm{I}}$. When $\Omega^{\mathrm{I}}$ is increased further still, the results (the ionic forces and $\rho^{\mathrm{I}}$ ) are found to change only very slightly. We note that regardless of the size of $\Omega^{\mathrm{I}}$, the total density is always found to be continuous at the boundary due to the high energy that the kinetic energy functional assigns to a discontinuity in the density. In Fig. 5 we have plotted the total charge density after minimizing with respect to $\rho^{\mathrm{I}}$, with $\rho^{\mathrm{II}}$ given by (a) a superposition of $\rho^{\text {at,gas }}$, and (b) a superposition of $\rho^{\text {at,cryst }}$. The particular slice of the charge density is a (100) plane that passes through one of the central atomic planes of the region I cluster. In (c) and (d) we have plotted the difference between these coupled charge densities and the density of this system when computed entirely with OF-DFT. In general, using $\rho^{\text {at,cryst }}$ results in a more accurate total charge density. From (c) and (d), it is clear that the superposition of $\rho^{\text {at,cryst }}$ reproduces the pure OF-DFT crystal charge density better than $\rho^{\text {at,gas }}$ both for points $\mathbf{r}$ well within $\Omega^{\mathrm{I}}$, as well as at the boundary of $\Omega^{\mathrm{I}}$.

It turns out, however, that the forces on the ions, for both choices of $\rho^{\text {at }}$, are comparable. Also comparable is the amount of deviation from the perfect lattice positions upon atomic relaxation for both choices. The exact numbers for these quantities for both coupling methods and the two choices of $\rho^{\text {at }}$ are summarized in Table I.

We have also applied this coupling method to study the core structure of the screw dislocation in aluminum. This system is a prime candidate for multiscale treatment, as the core structure depends strongly on both the atomic scale energetics, and on the long-range strain field of the dislocation. ${ }^{23}$

In aluminum the screw dislocation line lies in the [110] direction. The multiscale system considered here consists of a periodic structure along the dislocation line (modeling an infinite straight dislocation), with the minimal period along this direction, that is, two (110) planes. In the directions perpendicular to the dislocation line, open boundary conditions are imposed, consistent with continuum elasticity theory far from the dislocation core, as explained below. The dimensions of the region $\Omega^{\mathrm{I}}$ were chosen to be of $31.85 \times 31.85 \times 2.85 \AA$ in the $[1 \overline{1} 2],[1 \overline{1} \overline{1}]$, and [110] directions, respectively. This extends slightly beyond the region I atoms and into the region II atoms [see Fig. 6(b)]. The EAM region (region II) extends to a radius of $95 \AA$, and the atoms between a radius of 85 and $95 \AA$ are fixed at positions determined by the elastic strain field of a screw dislocation in a continuous anisotropic material, as calculated from continuum elasticity theory, using values for elastic moduli obtained from OF-DFT calculations for bulk aluminum. The geometry of this calculation is illustrated in Fig. 6(a).

The system was relaxed via the alternating-relaxation scheme of Sec. IV B. In Fig. 6(b) we give the differential displacement map of the resulting screw dislocation, which 


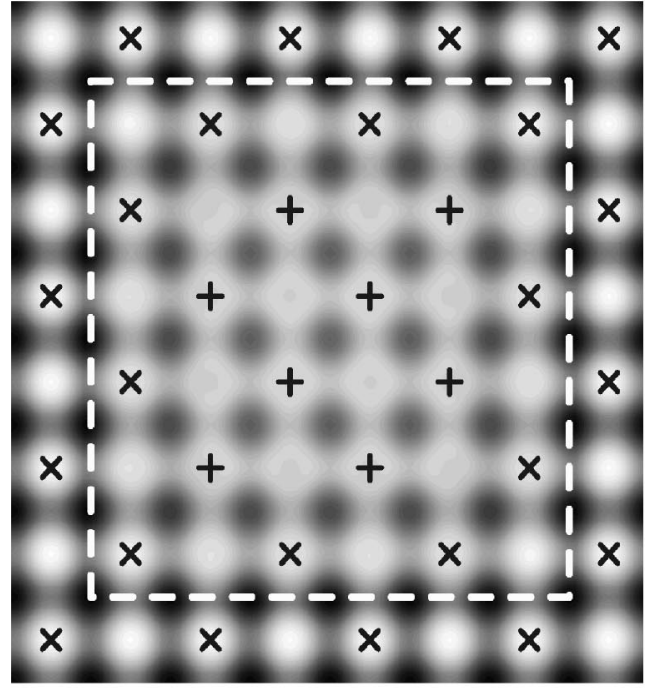

(a)

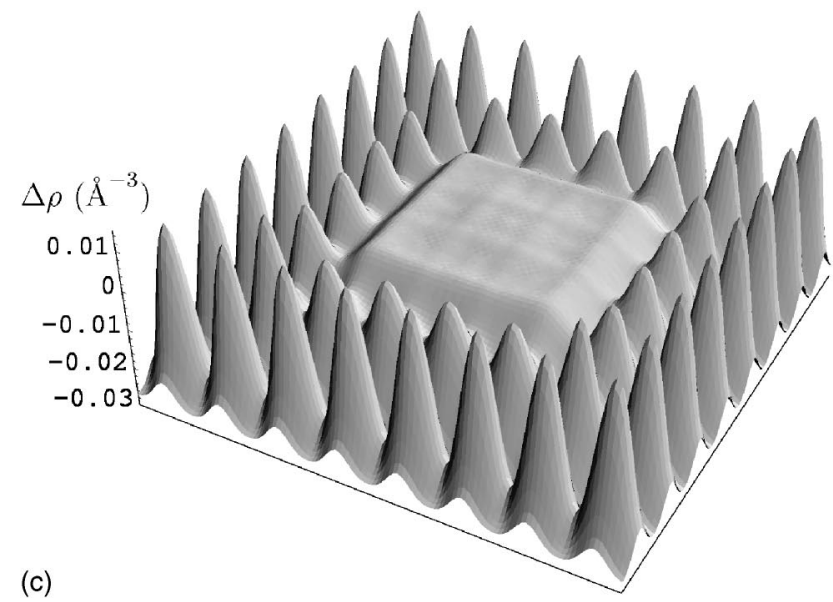

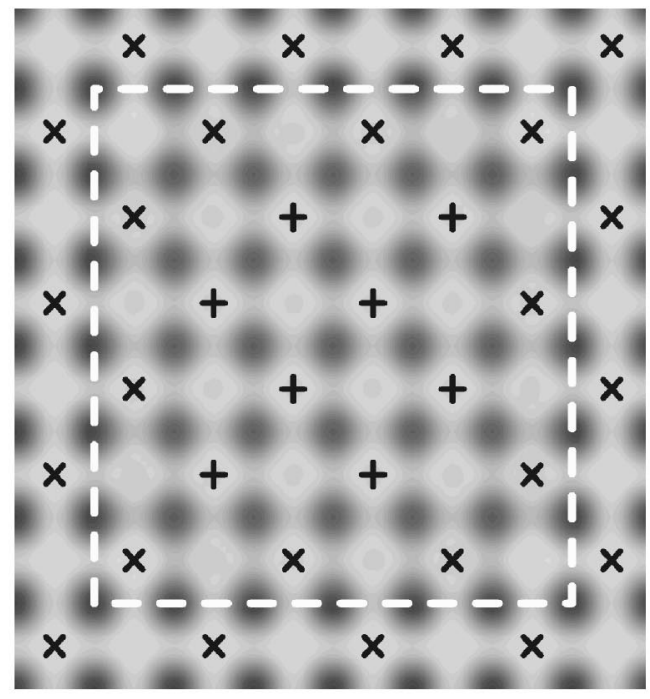

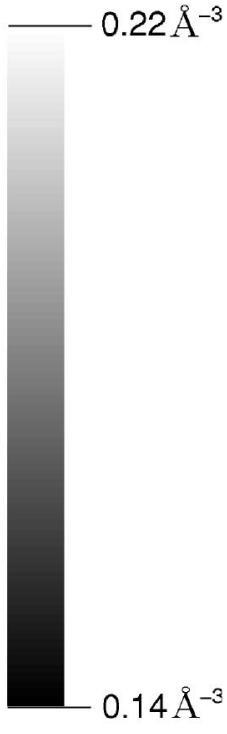

(b)

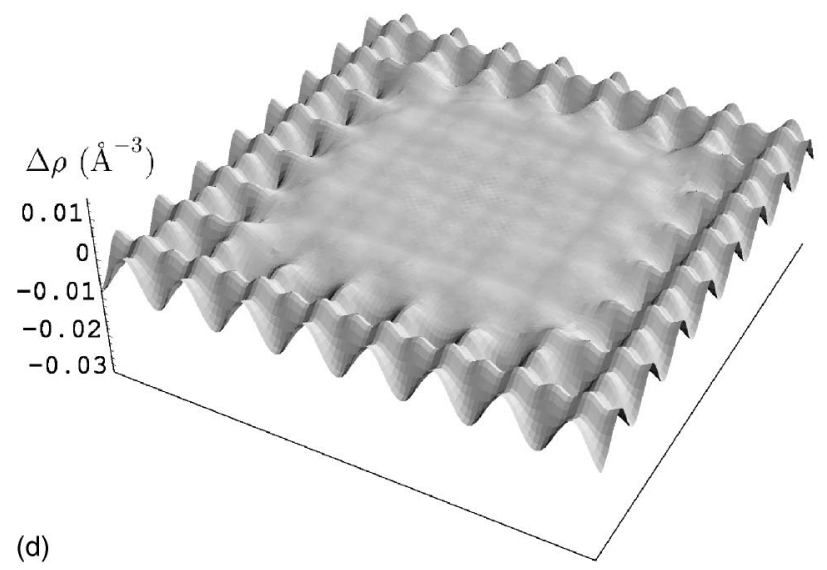

FIG. 5. Results for the quantum interaction energy method. (a) and (b) are contour plots of $\rho^{\text {tot }}$ with $\rho^{\text {II }}$ given by superpositions of $\rho^{\text {at,gas }}$ and $\rho^{\text {at,cryst }}$, respectively. The boundary of $\Omega^{\mathrm{I}}$ is shown with a dashed line, and the positions of the region I atoms lying in this plane are indicated by $(+)$, and region II atoms by $(\times)$. (c) and (d) show the difference between these two densities and the "correct" density coming from a purely OF-DFT calculation of the whole system.

shows the relative displacement along the [110] direction of neighboring [110] columns of atoms, modulo one Burgers vector. Figure 6(c) shows the relative displacement of the two $(1 \overline{11})$ planes that contain the dislocation, as a function of position along the $[1 \overline{1} 2]$ direction. For comparison, we also give this quantity for the screw dislocation as obtained using the EAM potential for the entire system. The screw dislocation as described by the coupling method is seen to have a wider extent and is more dissociated into partial dislocations than the dislocation described by the EAM potential. The

TABLE I. Summary of the performance of the two coupling methods, and the two choices for $\rho^{\text {at }}$ in the quantum interaction energy method. $F_{\max }^{\mathrm{I}}$ and $F_{\max }^{\mathrm{II}}$ are the maximum forces on region I atoms and region II atoms before relaxation and $F_{\text {av }}^{\mathrm{I}}$ is the average force on region I atoms before relaxation. $d_{\max }^{\mathrm{I}}$ and $d_{\max }^{\mathrm{II}}$ are the maximum displacements from the perfect lattice positions upon relaxation for region I and region II atoms and $d_{\mathrm{av}}^{\mathrm{I}}$ is the average displacement of region I atoms after relaxation.

\begin{tabular}{|c|c|c|c|c|c|c|}
\hline Interaction & $F_{\max }^{\mathrm{I}}$ & $\begin{array}{c}F_{\max }^{\mathrm{II}} \\
(\mathrm{eV} / \mathrm{A})\end{array}$ & $F_{\text {av }}^{\mathrm{I}}$ & $d_{\max }^{\mathrm{I}}$ & $\underset{(\AA)}{d_{\max }^{\mathrm{II}}}$ & $d_{\mathrm{av}}^{\mathrm{I}}$ \\
\hline Classical & 0.45 & 0 & 0.33 & 0.12 & 0.05 & 0.07 \\
\hline Quantum, $\rho^{\text {at,gas }}$ & 0.12 & 0.27 & 0.05 & 0.12 & 0.15 & 0.08 \\
\hline Quantum, $\rho^{\text {at,cyrst }}$ & 0.09 & 0.37 & 0.06 & 0.22 & 0.26 & 0.13 \\
\hline
\end{tabular}




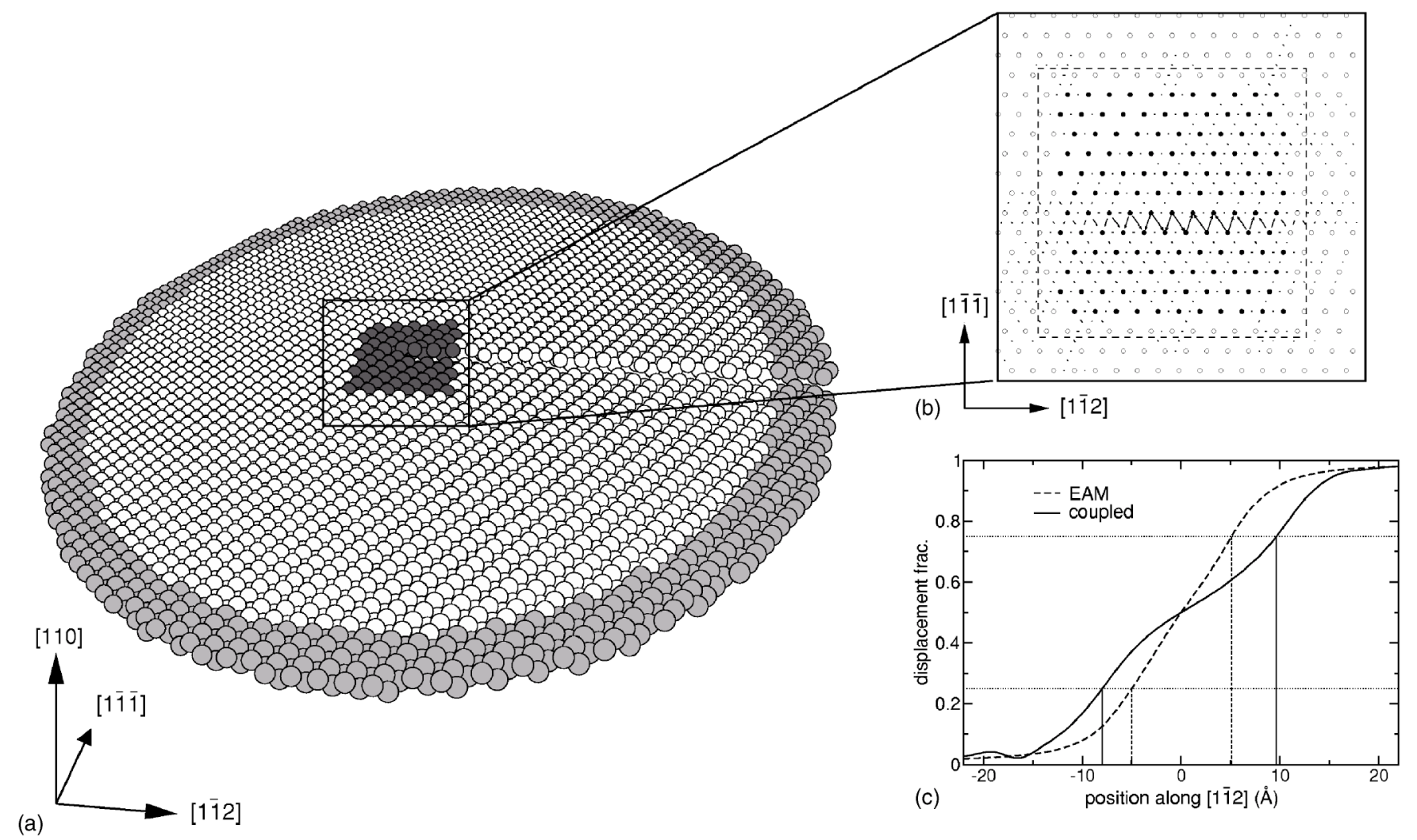

FIG. 6. (a) The multiscale system used to simulate the screw dislocation in aluminum. The dark atoms are OF-DFT atoms within region $\Omega^{\mathrm{I}}$, the white atoms are EAM atoms, and the gray atoms are EAM atoms that are fixed at positions determined by elasticity theory. (b) A differential displacement map of the dislocation core simulated with the multiscale method. The region $\Omega^{\mathrm{I}}$ is indicated with a dashed line. (c) The relative displacement along the [110] direction of the two (111) planes that contain the screw dislocation, for both the EAM simulation and the OF-DFT/EAM coupled simulation. The locations where the relative displacement equals $1 / 4$ and $3 / 4$ of the full Burgers-vector slip are indicated, defining the width of the dislocation core.

extent of the dislocation is determined by a balance between elastic energy, which tends to widen the dislocation, and the stacking fault energy, which tends to narrow the dislocation. The EAM potential was scaled such that it exhibits the same elastic properties as the OF-DFT method, but the two methods give different values for the stacking fault energies: the EAM potential gives a stacking fault energy of $105 \mathrm{~mJ} / \mathrm{m}^{2}$, while the OF-DFT stacking fault energy is lower, at $43 \mathrm{~mJ} / \mathrm{m}^{2}$. Thus, the multiscale simulation shows that the dislocation core structure is being determined by the energetics of the OF-DFT method in the core region, which allows the dislocation to widen. The amount of widening is quantified by comparing the regions between the locations where the displacement along the [110] direction reaches the values of one-quarter and three-quarters of the Burgers vector, as shown in Fig. 6(c). This is a clear and quantitative example of how the inclusion of the quantum-mechanical description in the dislocation core region changes the results of the simulation. To give a comparison of the efficiency of the coupling method, had the above calculation been done using OF-DFT to treat the entire system, the amount of computational time required would have been higher by a factor of 100 . For systems where region I is a smaller fraction of the entire system (which typically is the case for fully three- dimensional systems), the advantage of using the multiscale method is further enhanced.

\section{CONCLUSIONS}

The coupling of classical and quantum simulation in simple metals involves a set of challenges quite different than those for the coupling of covalently bonded materials and molecules, and hence requires a different set of approaches. We have presented here two methods for combining classical and quantum-mechanical simulation of simple metals. Both are based on a similar partitioning of the energy of the system, but they differ in how the energy of interaction between the classical and quantum-mechanical parts of the system are treated. We have presented numerical implementations that allow both coupling methods to be efficient.

Within the first coupling method, in which the interaction energy is determined from the classical potential, forces in the classical region are fully determined by the classical potential. Forces in the quantum region are determined by both classical and quantum energetics, the quantum energetics dominating well within the quantum region. A major practical advantage of this approach is that, if region I contains many different atomic species while region II 
contains only one atom type, there is no need for a classical potential for each species and their interactions; if the various species of atoms are well within region I, then the potential representing them does not matter at all as interactions in this region are treated purely with quantum mechanics.

Within the second coupling method, in which the interaction energy is determined via OF-DFT, forces in the classical region are determined mostly by the classical potential, with quantum contributions to atomic forces near the boundary of the regions. Forces in the quantum region are determined fully by quantum energetics. Within the quantum region, the charge density accurately reproduces the correct charge density, and smoothly joins with the implicit density (given by a sum of atomic densities) of the classical region.

Test results indicate that the second coupling method yields more accurate forces on the atoms in the quantum region than the first method, but that the first method yields more accurate forces for the atoms in the classical region. This may be due, to some extent, to the less accurate OF-DFT method used in the test of the second coupling method. The first coupling method also yielded a better relaxed

structure, probably due to its better treatment of forces on the classical atoms. However, unlike the first method, the second coupling method results in a more accurate charge density within the quantum-mechanical region, allowing for an accurate treatment of physical problems such as the introduction of impurities, where the background density is important. We also find that a superposition of atomic charge densities can reproduce the actual charge density well for a simple metallic system, given an appropriate choice for the atomic charge density; this allows for a smooth density transition at the boundary between regions.

Clearly, an important issue affecting the coupling quality for both methods is the agreement between forces from the DFT methods; within both methods there are atoms whose forces are determined by a combination of quantum and classical energetics, and the more closely the two energetics agree, the better the coupling will be. An improvement in the quality of the coupling might be obtained if the classical potential employed in region II is optimized to closely reproduce the DFT energetics; this is also in accord with the multiscale philosophy that a coupled simulation should act as if the most accurate method were used to simulate the entire system.

\section{ACKNOWLEDGMENTS}

The authors wish to thank Paul Maragakis, Ryan Barnett, Emily Carter, and William Curtin for fruitful discussions. This work was supported in part by a MURI-AFOSR Grant No. F49620-99-1-0272.

\section{APPENDIX: EVALUATING THE INTERACTION ENERGY FOR COMPLEX KINETIC ENERGY FUNCTIONALS}

We describe here how the interaction energy can be efficiently calculated when the approximate kinetic energy func- tional used is of a more complicated form, containing a convolution term of the form of Eq. (20). That is, we will describe a method for evaluating

$$
\begin{aligned}
T_{\mathrm{K}}^{\mathrm{int}}\left[\rho^{\mathrm{I}}, \rho^{\mathrm{II}}\right]= & \int f_{12}(\mathbf{r}) K\left(\mathbf{r}-\mathbf{r}^{\prime}\right) g_{12}\left(\mathbf{r}^{\prime}\right) d \mathbf{r} d \mathbf{r}^{\prime} \\
& -\int f_{1}(\mathbf{r}) K\left(\mathbf{r}-\mathbf{r}^{\prime}\right) g_{1}\left(\mathbf{r}^{\prime}\right) d \mathbf{r} d \mathbf{r}^{\prime} \\
& -\int f_{2}(\mathbf{r}) K\left(\mathbf{r}-\mathbf{r}^{\prime}\right) g_{2}\left(\mathbf{r}^{\prime}\right) d \mathbf{r} d \mathbf{r}^{\prime},
\end{aligned}
$$

where we have defined $f_{1}(\mathbf{r}) \equiv f\left(\rho^{\mathrm{I}}(\mathbf{r})\right), f_{12}(\mathbf{r}) \equiv f\left(\rho^{\mathrm{I}}(\mathbf{r})\right.$ $\left.+\rho^{\mathrm{II}}(\mathbf{r})\right)$, and so on. Then we define two new functions,

$$
\begin{gathered}
F(\mathbf{r}) \equiv f_{12}(\mathbf{r})-f_{2}(\mathbf{r}), \\
G(\mathbf{r}) \equiv g_{12}(\mathbf{r})-g_{2}(\mathbf{r}) .
\end{gathered}
$$

Note that $F(\mathbf{r})$ and $G(\mathbf{r})$ are zero for points $\mathbf{r} \notin \Omega^{\mathrm{I}}$. Using $F$ and $G$ we can reexpress Eq. (A1) as

$$
\begin{aligned}
T_{\mathrm{K}}^{\mathrm{int}}= & \int_{\Omega^{\mathrm{I}}} F(\mathbf{r})(K * G)(\mathbf{r}) d \mathbf{r}-\int_{\Omega^{\mathrm{I}}} f_{1}(\mathbf{r})\left(K * g_{1}\right)(\mathbf{r}) d \mathbf{r} \\
& +\int_{\Omega^{\mathrm{I}}} F(\mathbf{r})\left(K * g_{2}\right)(\mathbf{r}) d \mathbf{r}+\int_{\Omega^{\mathrm{I}}} G(\mathbf{r})\left(K * f_{2}\right)(\mathbf{r}) d \mathbf{r},
\end{aligned}
$$

where we have now defined

$$
(K * G)(\mathbf{r}) \equiv \int K\left(\mathbf{r}-\mathbf{r}^{\prime}\right) G\left(\mathbf{r}^{\prime}\right) d \mathbf{r}^{\prime},
$$

etc. We point out that if this interaction energy is being calculated in a coupled simulation in which the energetics of region I are calculated using the same kinetic energy (i.e., $E[\mathrm{I}]$ and $E^{\mathrm{int}}[\mathrm{I}, \mathrm{II}]$ being treated at the same level of theory), then the final term of Eq. (A3) is equal to and will cancel with the corresponding term in $E[\mathrm{I}]$.

So with Eq. (A3) we have expressed $T_{\mathrm{K}}^{\text {int }}$ purely in terms of intergrals over $\Omega^{\mathrm{I}}$; the problem is now reduced to efficiently calculating the functions $\left(K * f_{2}\right)(\mathbf{r})$ and $\left(K * g_{2}\right)$ $\times(\mathbf{r})$ for points $\mathbf{r}$ within $\Omega^{\mathrm{I}}$. A straightforward integration for each point $\mathbf{r} \in \Omega^{\mathrm{I}}$ is not an option, because $K(r)$ is typically long-ranged, and thus determining $\left(K * f_{2}\right)(\mathbf{r})$ at one single point $\mathbf{r}$ would require an integration over the volume of the whole coupled system, which would be highly inefficient. We now describe a method for determining $\left(K * f_{2}\right)(\mathbf{r})$, and $\left(K * g_{2}\right)(\mathbf{r})$ can be determined with precisely the same method.

In earlier work ${ }^{24}$ we have developed a method for efficiently evaluating convolutions such as Eq. (A4) when the convolution kernel $K(r)$ is of the particular form typically encountered in kinetic energy functionals involving convolution terms. ${ }^{16-20}$ We will invoke this method to determine $\left(K * f_{2}\right)$. In this method, the kernel is fitted in reciprocal space with the following form: 


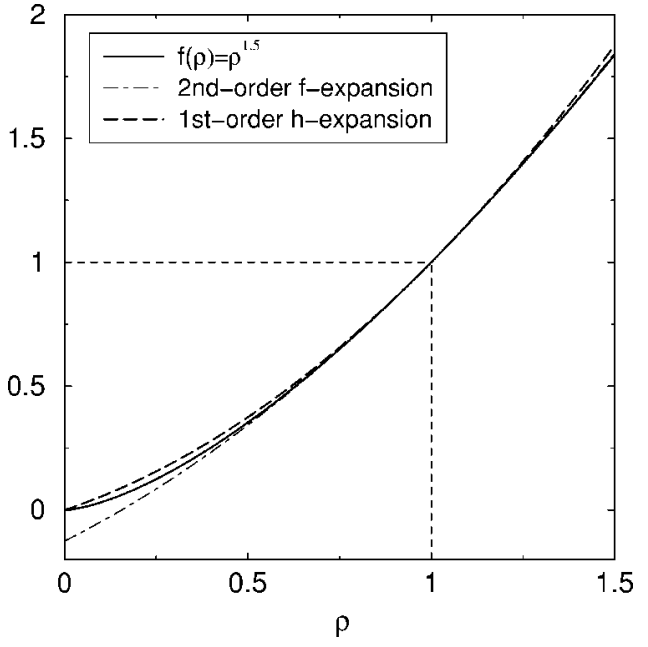

FIG. 7. A demonstration of the Taylor expansion of Eq. (A12) compared to a direct Taylor expansion of $f(\rho)=\rho^{1.5}$ about $\rho_{0}=1$.

$$
\begin{gathered}
\tilde{K}(k) \simeq \sum_{i} \tilde{K}_{i}(k), \\
\tilde{K}_{i}(k)=\frac{P_{i} k^{2}}{k^{2}+Q_{i}}
\end{gathered}
$$

where $P_{i}$ and $Q_{i}$ are complex fitting parameters. The kernels encountered in many kinetic energy functionals are well fit with this form, with four terms. The kernel in real space can be expressed as the sum of the inverse Fourier transform of each term of Eq. (A5):

$$
\begin{gathered}
K(\mathbf{r}) \simeq \sum_{i} K_{i}(\mathbf{r}), \\
K_{i}(\mathbf{r}) \equiv P_{i} \delta(\mathbf{r})-P_{i} Q_{i} \frac{e^{-\sqrt{Q_{i} r}}}{4 \pi r} .
\end{gathered}
$$

Thus $\left(K * f_{2}\right)$ can be written as the sum of separate convolutions:

$$
\begin{gathered}
\left(K * f_{2}\right)(\mathbf{r})=\sum_{i}\left(K_{i} * f_{2}\right)(\mathbf{r}) \\
\left(K_{i} * f_{2}\right)(\mathbf{r}) \equiv \int K_{i}\left(\mathbf{r}-\mathbf{r}^{\prime}\right) f_{2}\left(\mathbf{r}^{\prime}\right) d \mathbf{r}^{\prime} .
\end{gathered}
$$

Because in reciprocal space the $\left(K_{i} * f_{2}\right)$ satisfy

$$
\left[k^{2}+Q_{i}\right]\left(\widetilde{K_{i} * f_{2}}\right)(\mathbf{k})=P_{i} k^{2} \widetilde{f}_{2}(\mathbf{k}),
$$

in real space they satisfy

$$
\left[\nabla^{2}-Q_{i}\right]\left(K_{i} * f_{2}\right)(\mathbf{r})=P_{i} \nabla^{2} f_{2}(\mathbf{r}),
$$

i.e., they are solutions to (complex) Helmholtz equations that can be solved with conjugate-gradient-based methods, ${ }^{32}$ such methods are efficient and only involve operations within $\Omega^{\mathrm{I}}$. The solutions to Eqs. (A10) are only well defined when boundary conditions for $\left(K_{i} * f_{2}\right)(\mathbf{r})$ are supplied.
We propose the use of Dirichlet boundary conditions. The value of $\left(K_{i} * f_{2}\right)(\mathbf{r})$ for points $\mathbf{r}$ on the boundary of $\Omega^{\mathrm{I}}$ can be found by evaluating the convolutions, Eq. (A8). Because of the regular nature of $\rho^{\mathrm{II}}(\mathbf{r})$, being the sum of atomic densities, an efficient method for evaluating these convolutions exists. The form of the convolution that needs to be evaluated is

$$
\left(K_{i} * f_{2}\right)(\mathbf{r})=\int K_{i}\left(\mathbf{r}-\mathbf{r}^{\prime}\right) f\left(\sum_{j} \rho^{\mathrm{at}}\left(\mathbf{r}^{\prime}-\mathbf{R}_{j}^{\mathrm{II}}\right)\right) d \mathbf{r}^{\prime}
$$

If $f(\rho)$ were a linear function, then this would reduce to a sum of pair functions. For many kinetic energy functionals, $f(\rho)$ is not linear, but equal to some power of $\rho: f(\rho)=\rho^{\alpha}$. This leads us to consider a Taylor expansion of $f(\rho)$ about some average density $\rho_{0}$. This Taylor expansion suffers in places where $\rho^{\mathrm{II}}(\mathbf{r})$ is near 0 , which occurs, for instance, in the center of $\Omega^{\mathrm{I}}$. An expansion that is much more accurate down to small values of $\rho$ is obtained if we Taylor expand the function $h(\rho) \equiv f(\rho) / \rho$ and express $f\left(\rho^{\mathrm{II}}\right)$ in terms of this expansion:

$$
\begin{aligned}
f\left(\sum_{j} \rho^{\mathrm{at}}\left(\mathbf{r}^{\prime}-\mathbf{R}_{j}^{\mathrm{II}}\right)\right) \simeq & \left(\sum_{j} \rho^{\mathrm{at}}\left(\mathbf{r}^{\prime}-\mathbf{R}_{j}^{\mathrm{II}}\right)\right)\left[h\left(\rho_{0}\right)+h^{\prime}\left(\rho_{0}\right)\right. \\
& \left.\times\left[\sum_{k} \rho^{\mathrm{at}}\left(\mathbf{r}^{\prime}-\mathbf{R}_{k}^{\mathrm{II}}\right)-\rho_{0}\right]+\cdots\right] .
\end{aligned}
$$

In Fig. 7 we illustrate the effectiveness of relation (A12) compared to expanding $f(\rho)$ directly when $f(\rho)=\rho^{1.5}$ and $\rho_{0}=1$. Although the $h$ expansion is taken only to first order, while the $f$ expansion is taken to second order, the $h$ expansion is seen to be more accurate at small $\rho$.

Upon substitution of the expansion of Eq. (A12) in the convolution, Eq. (A11), we find

$$
\begin{aligned}
&\left(K_{i} * f_{2}\right)(\mathbf{r}) \simeq[\left.h\left(\rho_{0}\right)-\rho_{0} h^{\prime}\left(\rho_{0}\right)\right] \sum_{j} L_{i}^{(1)}\left(\mathbf{r}-\mathbf{R}_{j}^{\mathrm{II}}\right) \\
&+h^{\prime}\left(\rho_{0}\right) \sum_{j, k} L_{i}^{(2)}\left(\mathbf{r}-\mathbf{R}_{j}^{\mathrm{II}}, \mathbf{r}-\mathbf{R}_{k}^{\mathrm{II}}\right) \\
& L_{i}^{(1)}(\mathbf{R}) \equiv \int K_{i}\left(\mathbf{r}^{\prime}\right) \rho^{\mathrm{at}}\left(\mathbf{R}-\mathbf{r}^{\prime}\right) d \mathbf{r}^{\prime}, \\
& L_{i}^{(2)}\left(\mathbf{R}, \mathbf{R}^{\prime}\right) \equiv \int K_{i}\left(\mathbf{r}^{\prime}\right) \rho^{\mathrm{at}}\left(\mathbf{R}-\mathbf{r}^{\prime}\right) \rho^{\mathrm{at}}\left(\mathbf{R}^{\prime}-\mathbf{r}^{\prime}\right) d \mathbf{r}^{\prime}
\end{aligned}
$$

$L_{i}^{(1)}$ is the convolution of an atomic density with $K_{i}(r)$. $L_{i}^{(2)}\left(\mathbf{R}, \mathbf{R}^{\prime}\right)$ is the convolution of the product of two atomic densities with $K(r)$, and consequently vanishes when the two atomic densities do not overlap. The integrand is nonzero only where the overlap occurs. It thus makes sense to express $L_{i}^{(2)}$ in terms of new coordinates, illustrated in Fig. 8: 


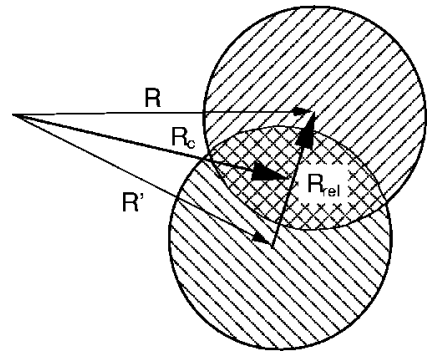

FIG. 8. New coordinates $\mathbf{R}_{\mathrm{c}}$ and $\mathbf{R}_{\text {rel }}$ for evaluating the threecenter integrals of $L_{i}^{(2)}$.

$$
M_{i}\left(\mathbf{R}_{\mathrm{c}}, \mathbf{R}_{\mathrm{rel}}\right) \equiv L_{i}^{(2)}\left(\mathbf{R}_{\mathrm{c}}-\frac{1}{2} \mathbf{R}_{\mathrm{rel}}, \mathbf{R}_{\mathrm{c}}+\frac{1}{2} \mathbf{R}_{\mathrm{rel}}\right) .
$$

In the case of a spherically symmetric $\rho^{\text {at }}(\mathbf{r}), L_{i}^{(1)}(\mathbf{R})$ is a function only of $|\mathbf{R}|$, and $M_{i}\left(\mathbf{R}_{\mathrm{c}}, \mathbf{R}_{\text {rel }}\right)$ will depend only on $\left|\mathbf{R}_{\mathrm{c}}\right|,\left|\mathbf{R}_{\text {rel }}\right|$, and $\mathbf{R}_{\mathrm{c}} \cdot \mathbf{R}_{\text {rel }}$. We now argue that the dependence on $\mathbf{R}_{\mathrm{c}} \cdot \mathbf{R}_{\mathrm{rel}}$ is weak. We can write the expression for $M_{i}$ as

$$
M_{i}\left(\mathbf{R}_{\mathrm{c}}, \mathbf{R}_{\mathrm{rel}}\right)=\int K_{i}\left(\mathbf{R}_{\mathrm{c}}-\mathbf{r}\right) \rho^{\mathrm{at}}\left(\mathbf{r}-\frac{1}{2} \mathbf{R}_{\mathrm{rel}}\right) \rho^{\mathrm{at}}\left(\mathbf{r}+\frac{1}{2} \mathbf{R}_{\mathrm{rel}}\right) d \mathbf{r} .
$$

If we expand $K$ about $\mathbf{R}_{\mathrm{c}}$, we find:

$$
\begin{aligned}
M_{i}\left(\mathbf{R}_{\mathrm{c}}, \mathbf{R}_{\mathrm{rel}}\right)= & \int\left[K_{i}\left(\left|\mathbf{R}_{\mathrm{c}}\right|\right)-\mathbf{r} \cdot \boldsymbol{\nabla} K_{i}\left(\mathbf{R}_{\mathrm{c}}\right)+\cdots\right] \\
& \times \rho^{\mathrm{at}}\left(\mathbf{r}-\frac{1}{2} \mathbf{R}_{\mathrm{rel}}\right) \rho^{\mathrm{at}}\left(\mathbf{r}+\frac{1}{2} \mathbf{R}_{\mathrm{rel}}\right) d \mathbf{r} .
\end{aligned}
$$

The integrals over the odd powers of $\mathbf{r}$ in the expansion of $K_{i}$ vanish by symmetry. Thus if we truncate the series at first order, the first-order term vanishes, leaving only the zeroth order term:

$$
\begin{gathered}
M_{i}\left(\mathbf{R}_{\mathrm{c}}, \mathbf{R}_{\mathrm{rel}}\right) \simeq K_{i}\left(\left|\mathbf{R}_{\mathrm{c}}\right|\right) P\left(\left|\mathbf{R}_{\mathrm{rel}}\right|\right), \\
P\left(\left|\mathbf{R}_{\mathrm{rel}}\right|\right) \equiv \int \rho^{\mathrm{at}}(\mathbf{r}) \rho^{\mathrm{at}}\left(\mathbf{r}-\mathbf{R}_{\mathrm{rel}}\right) d \mathbf{r} .
\end{gathered}
$$

Truncating the expansion of $K_{i}$ at the first order is reasonable, because $K_{i}(r)$ oscillates around the Fermi wavelength of the system, a length scale close to that of $\rho^{\text {at }}$. This approximate form, Eq. (A17) will behave quite badly at small $R_{\mathrm{c}}$, because $K_{i}$ diverges at the origin, and the radial averaging of this divergence that occurs in Eq. (A15) is not reflected in Eq. (A17). Thus we replace $K_{i}\left(\left|\mathbf{R}_{\mathrm{c}}\right|\right)$ there with the convolution of $K_{i}$ with a Gaussian of unit weight and a variance $r_{0}$ given roughly by the length scale of the overlap regions of the atomic densities [i.e., some fraction of the range of $\left.\rho^{\text {at }}(\mathbf{r})\right]$ :

$$
\begin{gathered}
M_{i}\left(\mathbf{R}_{\mathrm{c}}, \mathbf{R}_{\mathrm{rel}}\right) \simeq K_{i}^{\prime}\left(\left|\mathbf{R}_{\mathrm{c}}\right|\right) P\left(\left|\mathbf{R}_{\mathrm{rel}}\right|\right), \\
K_{i}^{\prime}(\mathbf{r}) \equiv\left(K_{i} * w\right)(\mathbf{r}), \\
w(\mathbf{r}) \equiv \pi^{-3 / 2} r_{0}^{-3} e^{-\left(r / r_{0}\right)^{2}} .
\end{gathered}
$$

Summarizing these results, we find that we can approximately evaluate $\left(K_{i} * f_{2}\right)$ as

$$
\begin{aligned}
\left(K_{i} * f_{2}\right)(\mathbf{r}) \simeq & {\left[h\left(\rho_{0}\right)-\rho_{0} h^{\prime}\left(\rho_{0}\right)\right] \sum_{j} L_{i}^{(1)}\left(\mathbf{r}-\mathbf{R}_{j}^{\mathrm{II}}\right) } \\
& +h^{\prime}\left(\rho_{0}\right) \sum_{\langle j, k\rangle} K_{i}^{\prime}\left(\left|\mathbf{r}-\frac{1}{2}\left(\mathbf{R}_{j}^{\mathrm{II}}+\mathbf{R}_{k}^{\mathrm{II}}\right)\right|\right) P\left(\left|\mathbf{R}_{j}^{\mathrm{II}}-\mathbf{R}_{k}^{\mathrm{II}}\right|\right),
\end{aligned}
$$

where the summation over $\langle j, k\rangle$ indicates that we need only sum over pairs of region II atoms with overlapping densities. The derivation of Eq. (A20) involved several approximations, and thus is not expected to be precise. We only propose that Eq. (A20) be used to generate the boundary conditions for Eq. (A10) that determine the $\left(K_{i} * f_{2}\right)(\mathbf{r})$ within region $\Omega^{\mathrm{I}}$, and we have found that the resulting $\left(K_{i} * f_{2}\right)(\mathbf{r})$ is more dependent on the source term than the boundary conditions. Nevertheless, because of the inaccuracies of Eq. (A20), we define a new region, $\Omega^{\mathrm{I}^{\prime}}$, that contains and extends a bit beyond $\Omega^{\mathrm{I}}$, and we use Eq. (A20) to obtain the boundary conditions for points $\mathbf{r}$ that lie on the boundary of $\Omega^{\mathrm{I}^{\prime}}$, and we solve Eqs. (A10) for all $\mathbf{r} \in \Omega^{\mathrm{I}^{\prime}}$, so that the resulting $\left(K_{i} * f_{2}\right)(\mathbf{r})$ are accurate for all $\mathbf{r} \in \Omega^{\mathrm{I}}$.

Thus we have all the pieces necessary to compute $T_{\mathrm{K}}^{\mathrm{int}}$. In summary, we do this as follows:

(a) Using Eq. (A20), we can evaluate $\left(K_{i} * f_{2}\right)(\mathbf{r})$ and $\left(K_{i} * g_{2}\right)(\mathbf{r})$ for points $\mathbf{r}$ on the boundary of a region $\Omega^{\mathrm{I}^{\prime}}$ that is slightly larger than $\Omega^{\mathrm{I}}$.

(b) Using those boundary conditions, the Helmholtz equations (A10) are solved, yielding $\left(K_{i} * f_{2}\right)(\mathbf{r})$ and $\left(K_{i} * g_{2}\right)(\mathbf{r})$ for all points $\mathbf{r} \in \Omega^{\mathrm{I}^{\prime}}$.

(c)Then $\left(K * f_{2}\right)(\mathbf{r})$ and $\left(K * g_{2}\right)(\mathbf{r})$ are constructed with Eq. (A7), and we can evaluate $T_{\mathrm{K}}^{\text {int }}$ via Eq. (A3).

Ther kernel interaction energy $T_{\mathrm{K}}^{\mathrm{int}}$ also gives a small contribution to the forces on region II atoms near the 1-2 boundary. By differentiating Eq. (A1), we find

$$
\begin{gathered}
\frac{\partial T_{\mathrm{K}}^{\mathrm{int}}}{\partial \mathbf{R}_{j}^{\mathrm{II}}}=-\int \boldsymbol{\nabla} \rho\left(\mathbf{r}-\mathbf{R}_{j}^{\mathrm{II}}\right)\left[f_{12}^{\prime}(\mathbf{r})(K * G)(\mathbf{r})+g_{12}^{\prime}(\mathbf{r})(K * F)(\mathbf{r})\right. \\
\left.+F^{\prime}(\mathbf{r})\left(K * g_{2}\right)(\mathbf{r})+G^{\prime}(\mathbf{r})\left(K * f_{2}\right)(\mathbf{r})\right] d \mathbf{r}, \\
(K * F)(\mathbf{r}) \equiv \int K\left(\mathbf{r}-\mathbf{r}^{\prime}\right) F\left(\mathbf{r}^{\prime}\right) d \mathbf{r}^{\prime}, \\
f_{12}^{\prime}(\mathbf{r}) \equiv f^{\prime}\left(\rho^{\mathrm{I}}(\mathbf{r})+\rho^{\mathrm{II}}(\mathbf{r})\right), \\
F^{\prime}(\mathbf{r}) \equiv f_{12}^{\prime}(\mathbf{r})-f^{\prime}\left(\rho^{\mathrm{II}}(\mathbf{r})\right),
\end{gathered}
$$

etc. 
*Electronic address: choly@fas.harvard.edu

${ }^{\dagger}$ Present address: Physics and Astronomy Department, California State University Northridge, Northridge, California 91330, USA.

${ }^{1} \mathrm{G}$. Lu and E. Kaxiras, in Handbook of Theoretical and Computational Nanotechnology, edited by M. Rieth and W. Schommers (American Scientific Publishers, Stevenson Ranch, CA, 2004), Chap. 22.

${ }^{2}$ W. E and Z. Y. Huang, J. Comput. Phys. 182, 234 (2002).

${ }^{3}$ W. Cai, M. de Koning, V. V. Bulatov, and S. Yip, Phys. Rev. Lett. 85, 3213 (2000).

${ }^{4}$ P. Hohenberg and W. Kohn, Phys. Rev. 136, B864 (1964).

${ }^{5}$ W. Kohn and L. J. Sham, Phys. Rev. 140, 1133 (1965).

${ }^{6} \mathrm{~J}$. Gao, in Reviews in Computational Chemistry, edited by K. B. Libkowitz and D. B. Boyd (VCH, New York, 1996), pp. 119185.

${ }^{7}$ N. Govind, Y. A. Wang, A. J. R. da Silva, and E. A. Carter, Chem. Phys. Lett. 295, 129 (1998).

${ }^{8}$ J. Q. Broughton, F. F. Abraham, N. Bernstein, and E. Kaxiras, Phys. Rev. B 60, 2391 (1999).

${ }^{9}$ Q. Cui, M. Elstner, E. Kaxiras, T. Frauenheim, and M. Karplus, J. Phys. Chem. B 105, 569 (2001).

${ }^{10}$ M. S. Daw, Phys. Rev. B 39, 7441 (1989).

${ }^{11}$ M. S. Daw and M. I. Baskes, Phys. Rev. B 29, 6443 (1984).

${ }^{12}$ F. Ercolessi, M. Parrinello, and E. Tosatti, Philos. Mag. A 58, 213 (1988).

${ }^{13}$ K. W. Jacobsen, J. K. Norskov, and M. J. Puska, Phys. Rev. B 35, 7423 (1987).

${ }^{14}$ T. A. Wesolowski and A. Warshel, J. Physiol. (London) 97, 8050 (1993).

${ }^{15}$ P. Cortona, Phys. Rev. B 44, 8454 (1991).
${ }^{16}$ E. Chacon, J. E. Alvarellos, and P. Tarazona, Phys. Rev. B 32, 7868 (1985).

${ }^{17}$ P. Garcia-Gonzalez, J. E. Alvarellos, and E. Chacon, Phys. Rev. B 53, 9509 (1996).

${ }^{18}$ L. W. Wang and M. P. Teter, Phys. Rev. B 45, 13196 (1992).

${ }^{19}$ Y. A. Wang, N. Govind, and E. A. Carter, Phys. Rev. B 58, 13465 (1998).

${ }^{20}$ Y. A. Wang, N. Govind, and E. A. Carter, Phys. Rev. B 60, 16350 (1999).

${ }^{21}$ T. Klüner, N. Govind, Y. A. Wang, and E. A. Carter, Phys. Rev. Lett. 88, 209702 (2002).

${ }^{22}$ F. Ercolessi and J. B. Adams, Europhys. Lett. 26, 583 (1994).

${ }^{23}$ D. Olmsted, K. Hardikar, and R. Phillips, Modell. Simul. Mater. Sci. Eng. 9, 215 (2001).

${ }^{24}$ N. Choly and E. Kaxiras, Solid State Commun. 121, 281 (2002).

${ }^{25}$ Y. A. Wang and E. A. Carter, in Theoretical Methods in Condensed Phase Chemistry, Progress in Theoretical Chemistry and Physics, edited by S. D. Schwartz (Kluwer, Dordrecht, The Netherlands, 2000), pp. 117-184.

${ }^{26}$ D. J. Gonzalez, L. E. Gonzalez, and M. J. Stott, Phys. Rev. Lett. 92, 085501 (2004).

${ }^{27}$ E. Smargiassi and P. A. Madden, Phys. Rev. B 49, 5220 (1994).

${ }^{28}$ B. J. Jesson and P. A. Madden, J. Chem. Phys. 113, 5935 (2000).

${ }^{29}$ D. C. Liu and J. Nocedal, Math. Program. 45, 503 (1989).

${ }^{30} \mathrm{H}$. Hellmann, Einfürung in die Quantenchemie (Deuticke, Leipzig, 1937).

${ }^{31}$ L. Goodwin, R. J. Needs, and V. Heine, J. Phys.: Condens. Matter 2, 351 (1990).

${ }^{32}$ R. W. Freund and N. M. Nachtigal, Numer. Math. 60, 315 (1991). 